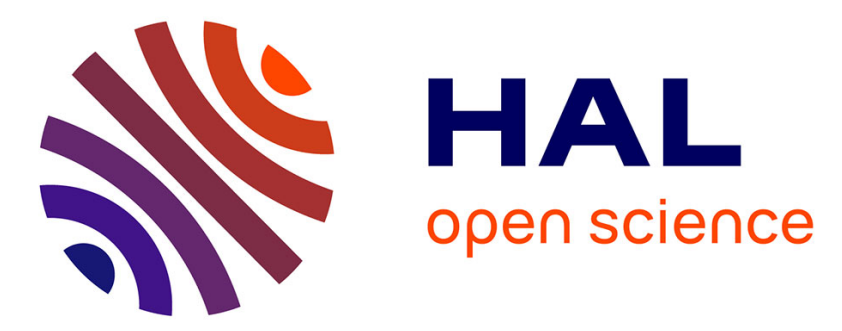

\title{
Transient growth of secondary instabilities in parallel wakes: Anti lift-up mechanism and hyperbolic instability
}

\author{
Sabine Ortiz, Jean-Marc Chomaz
}

\section{To cite this version:}

Sabine Ortiz, Jean-Marc Chomaz. Transient growth of secondary instabilities in parallel wakes: Anti lift-up mechanism and hyperbolic instability. Physics of Fluids, 2011, 23 (11), pp.114106 - 114106-15. 10.1063/1.3659158 . hal-00830701

HAL Id: hal-00830701

https://hal-ensta-paris.archives-ouvertes.fr/hal-00830701

Submitted on 8 Jul 2014

HAL is a multi-disciplinary open access archive for the deposit and dissemination of scientific research documents, whether they are published or not. The documents may come from teaching and research institutions in France or abroad, or from public or private research centers.
L'archive ouverte pluridisciplinaire HAL, est destinée au dépôt et à la diffusion de documents scientifiques de niveau recherche, publiés ou non, émanant des établissements d'enseignement et de recherche français ou étrangers, des laboratoires publics ou privés. 


\section{AIP $\mid \begin{aligned} & \text { Physics of } \\ & \text { Fluids }\end{aligned}$}

Transient growth of secondary instabilities in parallel wakes: Anti lift-up mechanism and hyperbolic instability

Sabine Ortiz and Jean-Marc Chomaz

Citation: Physics of Fluids (1994-present) 23, 114106 (2011); doi: 10.1063/1.3659158

View online: http://dx.doi.org/10.1063/1.3659158

View Table of Contents: http://scitation.aip.org/content/aip/journal/pof2/23/11?ver=pdfcov

Published by the AIP Publishing

\section{Articles you may be interested in}

Optimal perturbations of non-parallel wakes and their stabilizing effect on the global instability

Phys. Fluids 26, 024110 (2014); 10.1063/1.4866043

Stability of parallel wake flows in quasigeostrophic and frontal regimes

Phys. Fluids 18, 126602 (2006); 10.1063/1.2397563

A synthetic perturbative hypothesis for multiscale analysis of convective wake instability

Phys. Fluids 18, 054105 (2006); 10.1063/1.2201114

Lift-up process in a heated-cylinder wake flow

Phys. Fluids 18, 014106 (2006); 10.1063/1.2159031

Vortex shedding in high Reynolds number axisymmetric bluff-body wakes: Local linear instability and global bleed control

Phys. Fluids 16, 3460 (2004); 10.1063/1.1773071

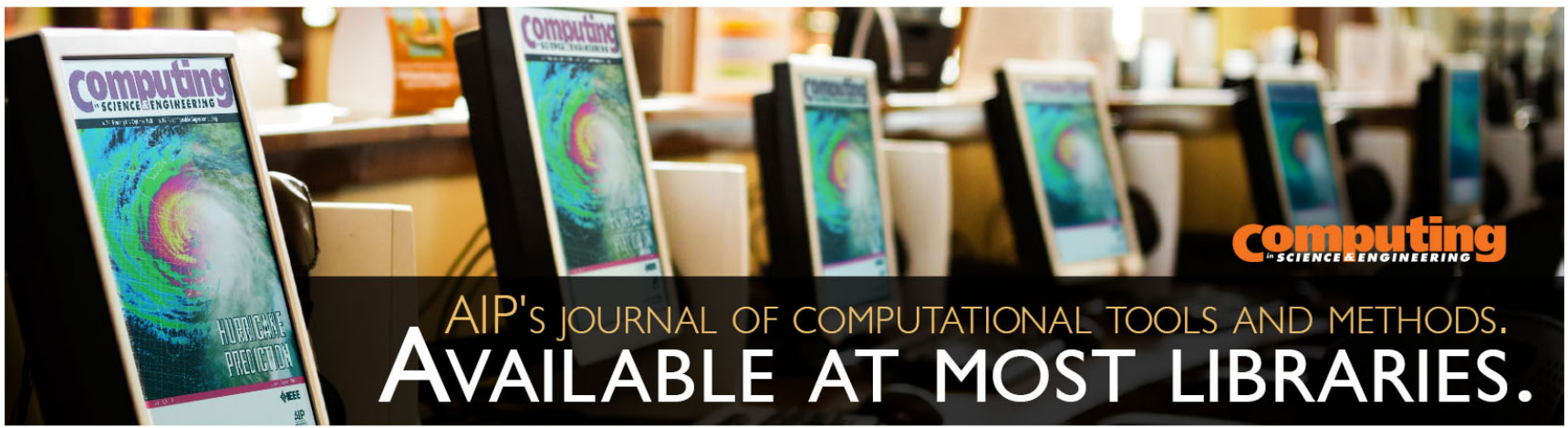




\title{
Transient growth of secondary instabilities in parallel wakes: Anti lift-up mechanism and hyperbolic instability
}

\author{
Sabine Ortiz ${ }^{1,2}$ and Jean-Marc Chomaz ${ }^{1}$ \\ ${ }^{1}$ LadHyX, CNRS-Ecole Polytechnique, F-91128 Palaiseau Cedex, France \\ ${ }^{2}$ UME/DFA, ENSTA-Paris Tech, chemin de la Hunière, 91761 Palaiseau Cedex, France
}

(Received 10 January 2011; accepted 14 October 2011; published online 28 November 2011)

This paper investigates the three-dimensional temporal instabilities and the transient growth of perturbations on a Von Kármán vortex street, issuing from the development of the primary instability of a parallel Bickley velocity profile typical of a wake forming behind a thin flat plate. By solving iteratively the linearized direct Navier Stokes equations and its adjoint equations, we compute the optimal perturbations that exhibit the largest transient growth of energy between the initial instant and different time horizons. At short time horizons, optimal initial perturbations are concentrated on the points of maximal strain of the base flow. The optimal gain of energy and the mechanism of instability are well predicted by local theories that describe the lagrangian evolution of a perturbation wave packet. At time of order unity, hyperbolic region leads the dynamics. Only at large time $(t \geq 20)$, the growth is led by the most amplified eigenmode. This eigenmode evolves, when the wavenumber increases, from perturbation centred in the core of the vortices, to perturbations localised on the stretching manifold of the hyperbolic points. At finite and large time, the gain in energy is initially associated with a mechanism reminiscent to the anti lift-up mechanism described by Antkowiak and Brancher [J. Fluid Mech. 578, 295 (2007)] in the context of an axisymmetric vortex. Presently, the optimal initial condition (the adjoint modes at large time) corresponding to streamwise streaks localised on the contracting manifold of the hyperbolic point induces streamwise vortices aligned with the stretching manifold of the hyperbolic point (the direct modes). The localisation on distinct manifolds of direct and adjoint eigenmodes is more pronounced when the Reynolds number is increased. An interpretation is proposed based on a balance between diffusion and stretching effects that predicts the thickness of the energy containing region for the adjoint and the direct mode decreasing as $1 / \sqrt{R e}$. The extra gain of energy due to non normal effects grows, since direct and adjoint modes are localised in different regions of space, i.e., the stretching and contracting manifold, a novel effect of the so called convective non normality associated with the transport of the perturbation by the base flow. (C) 2011 American Institute of Physics. [doi:10.1063/1.3659158]

\section{INTRODUCTION}

Wakes forming behind slender bodies are shear flow prototypes. Shear flows which present an initial velocity profile with an inflexion point are unstable to two-dimensional (2D) perturbations. As the primary Kelvin-Helmholtz instability ${ }^{1}$ develops, the initial vorticity rolls up leading to the formation of primary vortices transverse to the flow (spanwise). This primary state is itself unstable to 2D and three-dimensional secondary (3D) modes. The secondary two-dimensional instability induces the merging of two consecutive co-rotating vortices, involving the doubling of the initial primary wavelength. ${ }^{2}$ The secondary $3 \mathrm{D}$ instability is mainly characterized by the waviness of the primary vortices ${ }^{3}$ and the development of an array of counter-rotating vortices oriented in the direction of the flow (streamwise) which appears in the braid region between two consecutive primary vortices. In the case of a wake behind a flat plate, the primary instability of a wake leads to a double staggered rows of counter-rotating vortices transverse to the direction of the flow, the Von Kármán vortex street. The 3D secondary instability is then characterized by two different modes symmetric
(S) and anti-symmetric (AS), depending if they present the same or the opposite symmetries of the base flow as discussed by Robinson and $\mathrm{Saffman}^{4}$ and observed by Meiburg and Lasheras ${ }^{5}$ Lasheras and Meiburg. ${ }^{6}$ They were also identified and carefully studied in the wake of a cylinder (Williamson, ${ }^{7,8}$ Barkley and Henderson ${ }^{9}$ ) where they are labelled Mode A (symmetric mode) and Mode B (anti-symmetric mode). In this bluff body case, these secondary instabilities have been shown to be global $^{9}$ and bifurcations select a precise wavelength and involve all the region of the flow including the cylinder and the separation point. In slender body configuration, as the flat plate, both the primary and the secondary instabilities develop progressively in space and are extremely sensitive to perturbations added to the flow. They have been interpreted as the result of convective instability ${ }^{5,6,10}$ justifying the use of temporal instability to analyse their dynamics in a frame moving with the primary structure. In all the shear flows configurations, the precise nature of these secondary instabilities is not yet fully understood. In the case of mixing layers, two main physical mechanisms have been proposed to explain the development of the 3D secondary flows: the elliptic instability of the primary vortices 
(see the review article by Kerswell ${ }^{11}$ ) and the hyperbolic instability of the highly strained region between vortices. ${ }^{12-14}$ In the case of a wake behind a bluff body, the mechanism of instability leading to Mode A, which wavelength scales on the vortex core, has been associated with the elliptic instability and the Mode B which develops at an higher Reynolds number and have a shorter wavelength with hyperbolic instability. ${ }^{7,8}$ However, identification of the mechanism responsible for the symmetry and wavelength selection has been shown to be more complex in that case, since its global nature involves a complex feedback mechanism where velocity induction by vorticity perturbation and coupling with the separation region play a role. In the slender body case, this effect is weaker and if the classification in terms of symmetric and anti-symmetric modes is universal, the selection of the mode and of its wavelength is not. In particular, Julien et al. ${ }^{10}$ observed experimentally in the case of a wake behind a flat plate that both modes of instability have comparable wavelength of order of the wavelength of the two-dimensional vortex street and are the result of a same instability mechanism, as also shown by numerical temporal stability analysis. ${ }^{15,16}$

In this paper, a three dimensional secondary instability of a wake forming behind a thin flat plate has been addressed in the temporal framework at moderate and large Reynolds number for both symmetries. Transient growths of perturbations are studied by computing the finite time optimal perturbation that maximizes the energy growth at different time horizons with the direct-adjoint iterative technique used by Luchini. ${ }^{17}$ The role of the regions near the hyperbolic stagnation point and the point of maximum strain have been analysed. Mechanisms for transient amplification of energy are reminiscent, for short time horizon to the mechanism described by Caulfield and Kerswell ${ }^{14}$ for an inviscid perturbation of an uniform hyperbolic base flow, and for intermediate and long time horizons to a mechanism acting in an opposite way to the classical lift-up mechanism ${ }^{18}$ similar to the anti lift-up mechanism described by Antkowiak and Brancher ${ }^{19}$ for the transient growth of axisymmetric perturbation of an axisymmetric vortex. At long time horizon, the optimal perturbation (adjoint mode) and the long time response (direct mode) are localised respectively on the contracting and stretching manifold of the hyperbolic point. The localisation is more pronounced when the Reynolds number increases and Reynolds number dependency is related to the work of Lin and $\mathrm{Corcos}^{20}$ and $\mathrm{Neu}^{21}$ on the effect of viscous diffusion and stretching on secondary vortices. The extra gain of energy with respect to the Reynolds number is computed and its evolution analysed in the framework of the convective non normality introduced by Chomaz. ${ }^{22}$

\section{3D PERTURBATIONS OF A VON KÁRMÁN STREET}

The experiment of Julien et al. ${ }^{10}$ has shown that the velocity profile at the trailing edge of a thin flat plate is well fitted by a Bickley wake profile. The $2 \mathrm{D}$ wake was characterized by the Reynolds number, $R_{e}^{\exp }=\frac{\bar{U} \delta_{\omega}}{\nu_{\exp }}=220$ with $\bar{U}$ the free stream velocity, $\nu_{\exp }$ the fluid viscosity, $\delta_{\omega}$ the wake thickness, and by the Strouhal number $S_{t}=\delta_{\omega} f / \bar{U}$ $\sim \frac{2 \pi}{5} \frac{\delta_{\omega}}{\lambda_{2 D}} \sim 0.07$ with $f$ the frequency of the vortex shedding, and $\lambda_{2 D}$ the wavelength of the primary vortex; $\lambda_{2 D} \sim \frac{1}{5} \bar{U} f$ since the reported propagation velocity of the primary vortices forming the Von Kármán street is about $\frac{1}{5} \bar{U}$.

As in Ref. 16, we adopt the temporal framework well suited for this slowly developing wake. The same procedure is used to generate numerically a $2 \mathrm{D}$ base flow on which transient growth are analysed. Only the features useful for the present study will be recalled here. Since no exact solutions of the Navier-Stokes equations modelling a Von Kármán street are known, the base flow is obtained by a 2D direct numerical simulation of the wake resulting from the destabilization of a Bickley wake profile, which belongs to the family of velocity profiles used by Monkewitz ${ }^{23}$ to model bluff body wakes

$$
\mathbf{U}(y)=2-\frac{2}{1+\sinh ^{2}\left(\frac{y}{\delta}\right)},
$$

with $\delta=\ln (1+\sqrt{2})$. The velocity and length scales leading to the choice (1) in the numerical code are, respectively, $\bar{U} / 2$ and $\delta_{\omega} / \delta$. The symmetry of the Von Kármán street is imposed, and the diffusion of the Bickley wake is compensated by a body force. The $2 \mathrm{D}$ base state is computed with a pseudospectral method with an equally spaced Cartesian mesh of $128 \times 512$ collocation points. For convenience the x-length, $L_{x}$ is taken equal to $\lambda_{2 D}=2 \pi$ in order for the Strouhal number to match with the experimental value $S_{t}=0.07$. The crosstream length of the computational domain is $L_{y}=4 \lambda_{2 D}$, taken large enough to make confinement effects negligible. Periodic conditions are applied in the $\mathrm{x}$ and $\mathrm{y}$ directions. Fig. 1(a) presents the spanwise base vorticity $\Omega_{B}$ computed previously by Julien et al. ${ }^{16}$ The streamlines, in the Galilean frame which moves with the flow, are represented by arrowed lines on Fig. 1(a), its translation velocity being determined as in Ref. 16 by minimizing the modulus of the transport term $\left|\mathbf{U}_{\mathbf{B}} \cdot \nabla \mathbf{U}_{\mathbf{B}}\right|$ where $\mathbf{U}_{\mathbf{B}}$ is the base flow in the frame moving with the vortices. In that frame, the flow is quasi-steady with a slow evolution due to viscous diffusion. The streamlines possess two stagnation points, an hyperbolic point indicated by a white star and an elliptic point inside the vortex core indicated by a white triangle. The strain field associated with the base flow is plotted on Fig. 1(b).

$$
\epsilon(x, y)=\sqrt{\left(\frac{\partial v_{B}}{\partial y}\right)^{2}+\frac{1}{4}\left(\frac{\partial u_{B}}{\partial y}+\frac{\partial v_{B}}{\partial x}\right)^{2}},
$$

with $\left(u_{B}, v_{B}\right)$ the components of the velocity $\mathbf{U}_{\mathbf{B}}$ of the base flow in the $(x, y)$ plane. The location where maximum value of the strain, $\epsilon_{B}=0.4758$, is reached is marked by a white dot on Figs. 1(a) and 1(b). This point of maximum strain is close to the hyperbolic point (white star Fig. 1(b)), where the strain is slightly lower $\epsilon_{B}=0.41$.

For stability and transient growth analysis, the quasisteady base state $\mathbf{U}_{\mathbf{B}}$ in the frame moving with the vortices is used, the slow evolution of the base flow due to the viscous diffusion is neglected, and the base flow is kept constant. Infinitesimal 3D perturbation fields, with the same periodicity as the base flow, are superposed to this base state, of velocity $\mathbf{U}_{\mathbf{B}}$ and vorticity $\boldsymbol{\Omega}_{\mathbf{B}}$. The three dimensional perturbation of 
(a)

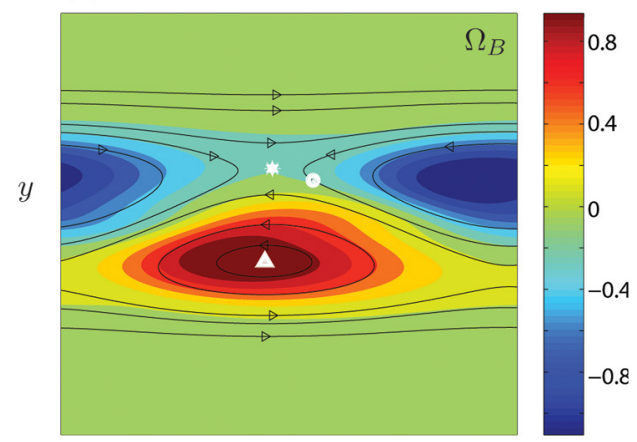

(b)

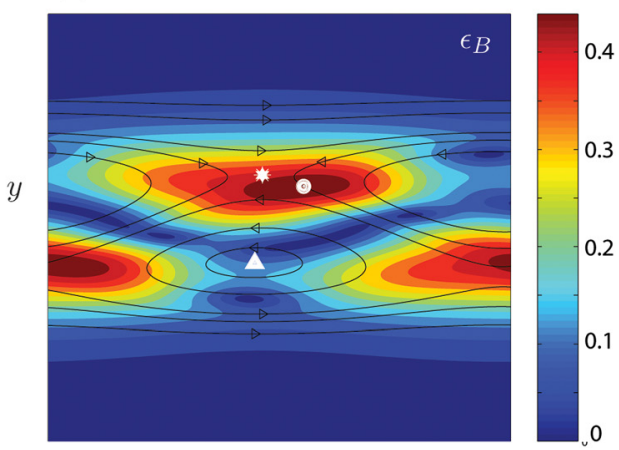

FIG. 1. (Color online) Isovalues of (a) the spanwise vorticity $\Omega_{\mathrm{B}}$ of the base state and (b) local strain $\epsilon_{B}$ in the (x,y) plane for $R_{e}=76.9$, Julien $e t$ al. ${ }^{16}$ The arrowed lines sketch the streamlines of the base flow in the frame the flow is quasi-steady. The computation is performed with $128 \times 512$ collocation points, on a computaional domain of size $\left(\mathrm{L}_{\mathrm{x}}, \mathrm{L}_{\mathrm{y}}\right)=(2 \pi, 8 \pi)$. The domain represented on (a) and (b) is $[0.24,2 \pi]$ in the $\mathrm{x}$ direction and [5.2, 20] in the $\mathrm{y}$ direction. The white star locates the hyperbolic stagnation point which has coordinates $(3.04,14.38)$ and the white triangle the elliptic stagnation point of coordinates $(2.94$, 11.34). The white point on (b) marked the location of the maximum of strain point of coordinates $(3.58,13.99)$.

velocity, vorticity, and pressure $[\tilde{\mathbf{U}}, \widetilde{\boldsymbol{\omega}}, \tilde{p}]$ are solutions of the linearized Navier-Stokes equations

$$
\left\{\begin{array}{l}
\frac{\partial \tilde{\mathbf{U}}}{\partial t}=\mathbf{U}_{B} \times \widetilde{\boldsymbol{\omega}}+\tilde{\mathbf{U}} \times \Omega_{\mathbf{B}}-\nabla\left(\tilde{p}+\tilde{\mathbf{U}} \cdot \mathbf{U}_{\mathbf{B}}\right)+\frac{1}{R_{e}} \Delta \tilde{\mathbf{U}} \\
\nabla \cdot \tilde{\mathbf{U}}=0
\end{array}\right.
$$

where viscous diffusion of the perturbation are taken into account. Note that with the present definition of the base flow, the numerical Reynolds number is $R_{e}=R_{e}^{\exp } /(2 \delta)$. The uniformity of the base state along the spanwise direction, $z$ axis, allows a decomposition of the perturbations on the form

$$
[\widetilde{\mathbf{U}}, \widetilde{\boldsymbol{\omega}}, \tilde{p}](\mathrm{x}, \mathrm{y}, \mathrm{z}, \mathrm{t})=[\mathbf{U}, \boldsymbol{\omega}, \mathrm{p}](\mathrm{x}, \mathrm{y}, \mathrm{t}) \mathrm{e}^{\mathrm{ik}_{\mathrm{z}} \mathrm{z}}+\mathrm{cc},
$$

where $\mathbf{U}=\left(\mathrm{u}_{\mathrm{x}}, \mathrm{u}_{\mathrm{y}}, \mathrm{u}_{\mathrm{z}}\right)$ is the velocity, $\boldsymbol{\omega}=\left(\omega_{x}, \omega_{y}, \omega_{z}\right)$ the vorticity, and $p$ the pressure of the perturbation associated with $\mathrm{k}_{\mathrm{z}}$ the spanwise wavenumber, and c.c. denotes the complex conjugate in Eq. (4). Since the base flow is symmetric under $\left(x, y, \Omega_{B}\right) \rightarrow\left(x+\lambda_{2 D} / 2,-y,-\Omega_{B}\right)$, the 3D secondary perturbation may be decomposed as the sum of a $\mathrm{S}$ and AS part, which evolve independently when non linearities are not taken into account.

The symmetric contribution verifies

$$
\left\{\begin{array}{l}
{\left[\mathrm{u}_{\mathrm{x}}, \mathrm{u}_{\mathrm{y}}, \mathrm{u}_{\mathrm{z}}\right](\mathrm{x}, \mathrm{y}, \mathrm{t})=\left[\mathrm{u}_{\mathrm{x}},-\mathrm{u}_{\mathrm{y}}, \mathrm{u}_{\mathrm{z}}\right]\left(\mathrm{x}+\lambda_{2 \mathrm{D}} / 2,-\mathrm{y}, \mathrm{t}\right)} \\
{\left[\omega_{\mathrm{x}}, \omega_{\mathrm{y}}, \omega_{\mathrm{z}}\right](\mathrm{x}, \mathrm{y}, \mathrm{t})=\left[-\omega_{\mathrm{x}}, \omega_{\mathrm{y}},-\omega_{\mathrm{z}}\right]\left(\mathrm{x}+\lambda_{2 \mathrm{D}} / 2,-\mathrm{y}, \mathrm{t}\right)}
\end{array} .\right.
$$

The anti-symmetric contribution satisfies opposite relations

$$
\left\{\begin{array}{l}
{\left[\mathrm{u}_{\mathrm{x}}, \mathrm{u}_{\mathrm{y}}, \mathrm{u}_{\mathrm{z}}\right](\mathrm{x}, \mathrm{y}, \mathrm{t})=\left[-\mathrm{u}_{\mathrm{x}}, \mathrm{u}_{\mathrm{y}},-\mathrm{u}_{\mathrm{z}}\right]\left(\mathrm{x}+\lambda_{2 \mathrm{D}} / 2,-\mathrm{y}, \mathrm{t}\right)} \\
{\left[\omega_{\mathrm{x}}, \omega_{\mathrm{y}}, \omega_{\mathrm{z}}\right](\mathrm{x}, \mathrm{y}, \mathrm{t})=\left[\omega_{\mathrm{x}},-\omega_{\mathrm{y}}, \omega_{\mathrm{z}}\right]\left(\mathrm{x}+\lambda_{2 \mathrm{D}} / 2,-\mathrm{y}, \mathrm{t}\right)}
\end{array} .\right.
$$

The numerical integration of Eqs. (3) and (4) is based on a pseudospectral code ${ }^{24}$ in cartesian coordinates with periodic conditions, setting the transverse wavenumber $\mathrm{k}_{\mathrm{z}}$ constant during the simulation. Two sets of simulations have been performed for symmetric and anti-symmetric perturbations imposed on the initial conditions. The velocity, vorticity, and pressure perturbations are expressed in Fourier space by application of the complex Fourier transform

$$
[\mathbf{U}, \boldsymbol{\omega}, p](x, y, t)=\iint[\hat{\mathbf{U}}, \hat{\boldsymbol{\omega}}, \hat{p}]\left(k_{x}, k_{y}, t\right) e^{i\left(k_{x} x+k_{y} y\right)} d k_{x} d k_{y} .
$$

In spectral space, the linearized Navier-Stokes Equations (3) become

$$
\frac{\partial \hat{\mathbf{U}}}{\partial t}=\mathbf{P}(\mathbf{k})\left[\mathbf{U}_{B} \times \omega \widehat{+\mathbf{U}} \times \Omega_{B}\right]-\frac{1}{R_{e}} \mathbf{k}^{2} \hat{\mathbf{U}},
$$

where $\mathbf{k}=\left(k_{x}, k_{y}, \mathrm{k}_{\mathrm{z}}\right)$ is the total wavevector and $\mathbf{P}(\mathbf{k})$ is the projection operator on the space of divergence-free fields which, in Fourier space, may be expressed as a tensor with components $P_{i j}=\delta_{i j}-k_{i} k_{j} / \mathbf{k}^{2}$. Introduction of this operator suppresses the term $\nabla\left(\tilde{p}+\tilde{\mathbf{U}} \cdot \mathbf{U}_{B}\right)$. Time integration is performed on the Fourier space (i.e., on Eq. (8)) with a secondorder Adams-Bashforth scheme whereas the dissipative term $\frac{1}{R_{e}} \Delta \mathbf{U}$ is integrated exactly in the Fourier space. In the present pseudo spectral method, the cross-product terms $\mathbf{U}_{B} \times \boldsymbol{\omega}+\mathbf{U} \times \boldsymbol{\Omega}_{B}$ are evaluated in the physical space and projected back in the Fourier space. When searching for eigenmodes, Arnoldi technique based on a fifth dimensional Krylov space ${ }^{25}$ is implemented and allows us to retrieve with a reasonable accuracy the three leading modes. It should be noticed that for both symmetries, the leading modes are stationary $\left(\sigma_{i}=0\right)$ but less unstable propagating modes have also been identified. ${ }^{16,26}$ For $R_{e}=76.9\left(R_{e}^{\text {exp }}=220\right)$ and for the base flow of Fig. 1, Julien et al. ${ }^{16}$ have computed numerically the growth rate of the most amplified anti-symmetric and symmetric modes, which are stationary and represented on Fig. 2, respectively, by a solid line and a dash-dotted line. Using the same procedure, we have calculated, for both symmetries, the growth rate of amplified modes developing on the same base flow, but for a larger Reynolds number $R_{e}=769$, their growth rates $\sigma_{r}$ are plotted on Fig. 2 .

For $R_{e}=769$, the most amplified symmetric mode ("o" symbol) corresponds to a spanwise wavenumber $\mathrm{k}_{\mathrm{z}}=3.4$ with a growth rate $\sigma_{r}=0.141$. The band of unstable wavenumbers is $[0.18,12.5]$. For the anti-symmetric mode ("+" symbol), the maximum of amplification $\sigma_{r}=0.142$ is obtained for $\mathrm{k}_{\mathrm{z}}=2.8$ and the band of unstable wavenumbers is smaller $[0.36,11.53]$. The eigenmodes for the symmetric 


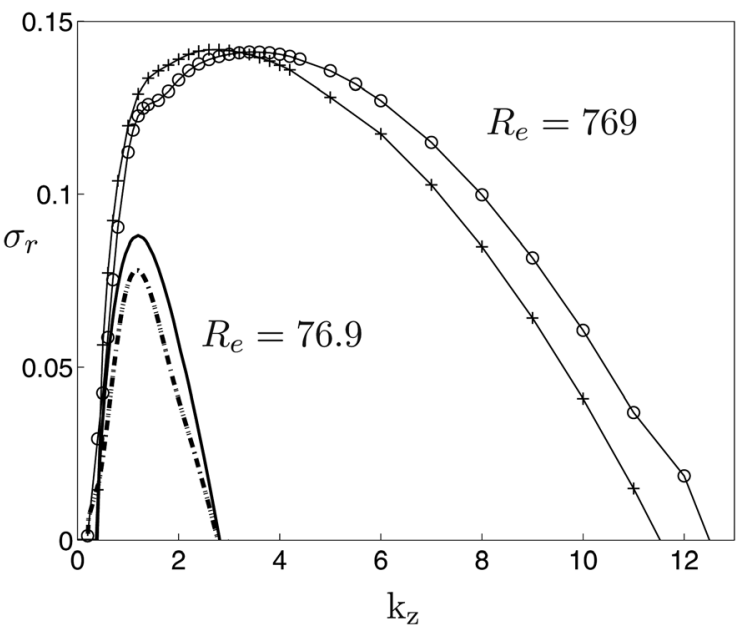

FIG. 2. Non dimensional growth rate $\sigma_{r}$ of the most amplified modes as a function of the spanwise wavenumber $\mathrm{k}_{\mathrm{z}}$. These modes are stationary $\left(\sigma_{\mathrm{i}}=0\right)$. Most amplified anti-symmetric mode for $R_{e}=76.9$ continuous line and for $\mathrm{R}_{\mathrm{e}}=769(+)$ symbols. Most amplified symmetric mode for $\mathrm{R}_{\mathrm{e}}=76.9$ dash-dotted line and for $\mathrm{R}_{\mathrm{e}}=769$ (o) symbols.

and anti-symmetric branches present similar structure, and we restrict the analysis to symmetric modes. Fig. 3 shows the spanwise vorticity $\omega_{z}$, the enstrophy $|\omega|^{2}$, and the kinetic energy $K=\left|U^{2}\right|$ in the $(x, y)$ plane for three spanwise wavenumbers $\mathrm{k}_{\mathrm{z}}=1, \mathrm{k}_{\mathrm{z}}=1.4$, and $\mathrm{k}_{\mathrm{z}}=3.4$ (leading spanwise wavenumber). For $\mathrm{k}_{\mathrm{z}}=1$, the spanwise vorticity presents a dipolar structure in the base vortex core (Fig. 3(a)). The enstrophy is intense on the stretching manifold of the hyperbolic point marked by a star on Fig. 3(b). However, at this low wavenumber, the region of the elliptic point marked by a triangle is where the kinetic energy is maximum (Fig. 3(c)). When the spanwise wavenumber is increased to $\mathrm{k}_{\mathrm{z}}=1.4$ and $\mathrm{k}_{\mathrm{z}}=3.4$, the dipolar structure of the spanwise vorticity of the $\mathrm{k}_{\mathrm{z}}=1$ case (Fig. 3(a)) is progressively transformed, becoming intense at the periphery of the vortex core for $\mathrm{k}_{\mathrm{z}}=3.4$ Fig. 3(g). In parallel, the energy fields become braid-centred with negligible contributions in the vortex core Figs. 3(e), 3(f), 3(h), and 3(i).

This behaviour is similar to the one found by Potylitsin and Peltier ${ }^{27}$ for the three dimensional instability of a twodimensional single vortex row, issuing from a shear layer after the roll up of Kelvin-Helmoltz billows and their pairing. They have observed that the model structure of eigenmodes evolves from core-centred to braid-centred when the wavenumber is increased. The localisation of the eigenmode energy on the stretching manifold of the hyperbolic point suggests that the physical origin may be the hyperbolic instability studied by Caulfield and Kerswell ${ }^{14}$ for a pure inviscid extensional flow predicting a growth rate of the perturbation equal to $\sigma_{l o c}=\sqrt{\epsilon_{B}^{2}-\Omega_{B}^{2} / 4}$. The corresponding viscous cutoff wavenumber is given by $\mathrm{k}_{c}=\sqrt{R_{e} \cdot \sigma_{l o c}} \cdot{ }^{28} \mathrm{With} \epsilon_{B}$ and $\Omega_{B}$ evaluated at the hyperbolic point, the value predicted by the local theory is $\sigma_{l o c}=0.41$ three times larger than the computed leading growth rate and the predicted neutral wavenumber $\mathrm{k}_{c}=18.550 \%$ higher than the computed one $\mathrm{k}_{c}=12.5$. However, the prediction is only valid for an uniform strain and it does not take into account the spatial inhomogeneity of the flow. Yet at short time, when the perturbation has not explored the complex structure of the base flow, one may imagine that the large amplification by the stretching of the perturbation at the hyperbolic point should hold for some time. As shown by Caulfield and Kerswell ${ }^{14}$, hyperbolic regions may be responsible for strong transient growth. In order to get a better understanding of the role of the hyperbolic stagnation point and of the complex flow structure in the dynamics, we investigate the transient growth restricting ourselves first to the leading spanwise wavenumber $\mathrm{k}_{\mathrm{z}}=3.4$ and to the symmetric mode.

\section{TRANSIENT GROWTH ANALYSIS}

\section{A. Large time dynamics}

To describe amplification of perturbation at finite time, we need to determine the optimal initial condition that maximizes the energy gain $\mathrm{G}(\tau)$ at each time horizon $\tau$

$$
\mathrm{G}(\tau)=\frac{E(\tau)}{E(0)}=\frac{(\tilde{\mathbf{U}}(\tau) \mid \tilde{\mathbf{U}}(\tau))}{(\tilde{\mathbf{U}}(0) \mid \tilde{\mathbf{U}}(0))},
$$

with $E(0)$ the initial kinetic energy of the perturbation and $E(\tau)$ its value at time $\tau(E(\tau)$ is the result of the integration of $K=\left|\mathbf{U}^{2}\right|$ Figs. 3(c), 3(f), or 3(i) over the numerical domain)

$$
E(\tau)=(\tilde{\mathbf{U}} \mid \tilde{\mathbf{U}})=\int_{0}^{L_{x}} \int_{0}^{L_{y}}\left(\tilde{\mathbf{U}}^{* T} \cdot \tilde{\mathbf{U}}\right) \mathrm{dxdy}
$$

the superscripts ${ }^{*}$ and ${ }^{T}$ denote the complex conjugate and the transposition and $L_{x}$ and $L_{y}$ correspond to the integration domain size in the $x$ and $y$ direction defined in Sec. II.

We consider the following inner product:

$$
\begin{aligned}
\left\langle\mathbf{f}^{\prime} \mid \mathbf{f}\right\rangle & =\int_{0}^{\tau} \int_{0}^{L_{x}} \int_{0}^{L_{y}} \mathbf{f}^{\prime * T} \cdot \mathbf{f} \mathrm{d} x \mathrm{dydt} \\
& =\int_{0}^{\tau} \int_{0}^{L_{x}} \int_{0}^{L_{y}}\left(\tilde{\mathbf{U}}^{\prime * T} \cdot \tilde{\mathbf{U}}+\tilde{p}^{* *} \tilde{p}\right) \mathrm{dxdydt}
\end{aligned}
$$

where $\mathbf{f}^{\prime}=\left(\tilde{\mathbf{U}}^{\prime}, \tilde{p}^{\prime}\right)$ and $\mathbf{f}=(\tilde{\mathbf{U}}, \tilde{p})$ are two complex state vectors and $\tau$ a prescribed arbitrary time. The adjoint linearized Navier-Stokes equations are derived using the Lagrange identity $^{29}$

$$
\left\{\begin{array}{l}
-\frac{\partial \tilde{\mathbf{U}}^{+}}{\partial t}=\Omega_{\mathbf{B}} \times \tilde{\mathbf{U}}^{+}-\nabla \times\left(\mathbf{U}_{\mathbf{B}} \times \tilde{\mathbf{U}}^{+}\right)-\nabla \tilde{p}^{+}+\frac{1}{R_{e}} \Delta \tilde{\mathbf{U}}^{+} \\
\nabla \cdot \tilde{\mathbf{U}}^{+}=0
\end{array}\right.
$$

The optimization condition will require the adjoint system (12) be marched backwards in time setting $t^{\prime}=-t$ allowing (12) to become well-posed; $\left[\tilde{\mathbf{U}}^{+}, \tilde{p}^{+}\right](x, y, z, t)$ are the adjoint velocity and pressure perturbations. The numerical integration of Eq. (12) is based on a pseudo-spectral technique, similar to the one used to solve the direct equation, with periodic conditions. The size of the box and the timestep are the same as those described in the direct case for the integration of Eq. (3). We focus first, on the symmetric adjoint eigenmode, for the most unstable spanwise wavenumber $\mathrm{k}_{\mathrm{z}}=3.4$. The same Arnoldi technique on a five dimension 
(a)

Spanwise Vorticity

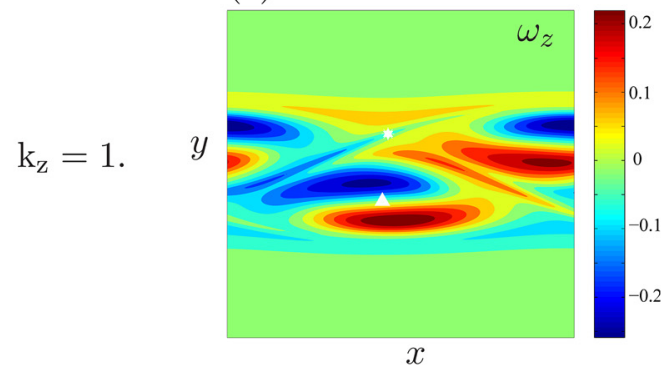

(d)

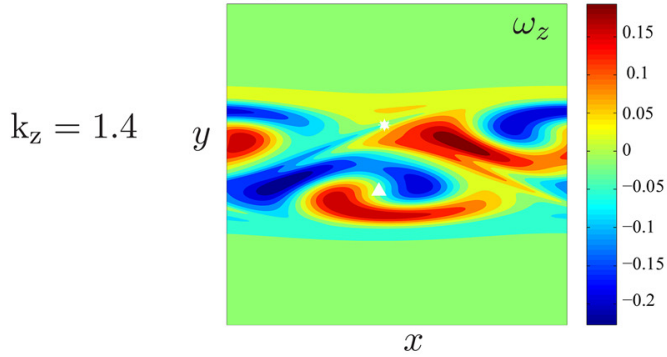

(g)

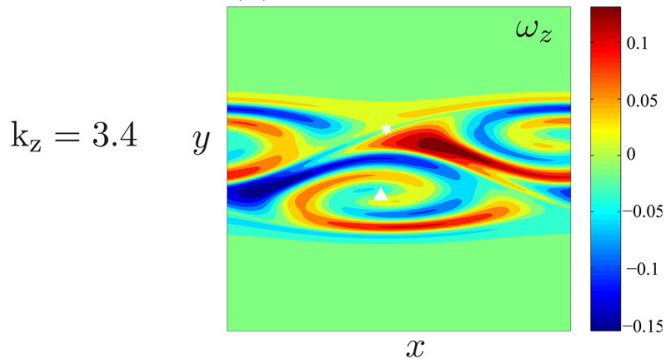

Enstrophy

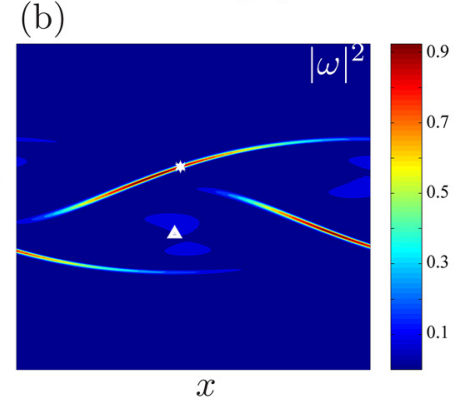

(e)

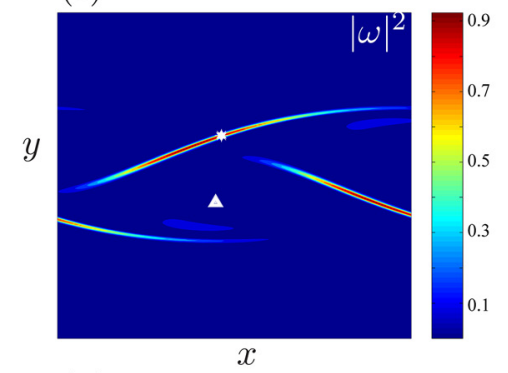

(h)

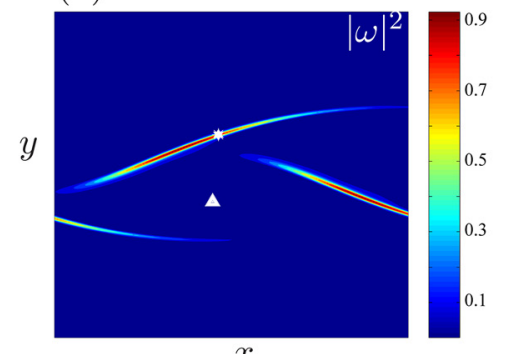

Kinetic Energy

(c)

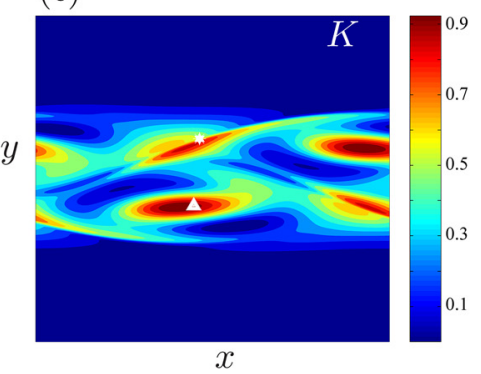

(f)

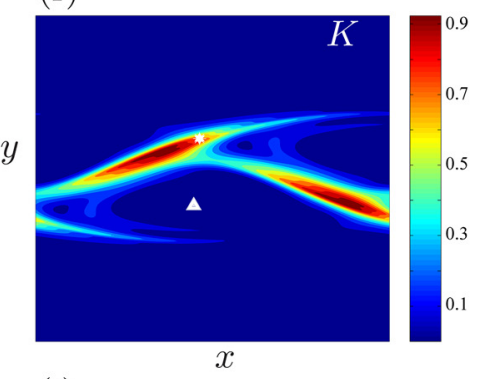

(i)

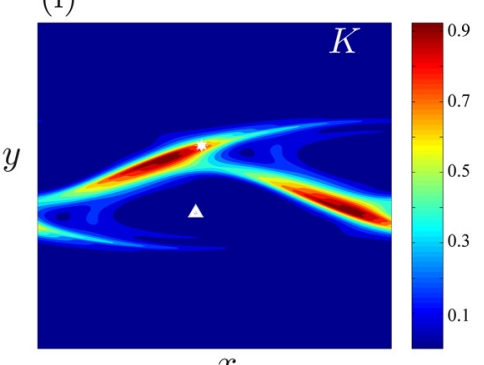

FIG. 3. (Color online) Symmetric mode: Spanwise vorticity field $\omega_{\mathrm{z}}(\mathrm{a}),(\mathrm{d})$, and (g), the enstrophy $|\omega|^{2}$ field (b), (e), and (h), and kinetic energy $\mathrm{K}=\left|\mathrm{U}^{2}\right|$ field (c), (f), and (i) associated with the eigenmode in the (x,y) plane for $\mathrm{R}_{\mathrm{e}}=769$ and for different wavenumbers (a)-(c) $\mathrm{k}_{\mathrm{z}}=1$; (d) $-(\mathrm{f}) \mathrm{k}_{\mathrm{z}}=1.4$; and (g)-(i) $\mathrm{k}_{\mathrm{z}}=3.4$. The spanwise vorticity has been scaled by $\mid \omega_{\max } l$, the square root of the maximum of the enstrophy, whereas enstrophy and energy fields have been normalized by their maximum amplitude. The domain represented is $[0.24,2 \pi]$ in the $\mathrm{x}$ direction and [5.2, 20] in the $\mathrm{y}$ direction. The white star locates the hyperbolic stagnation point which has coordinates $(3.04,14.38)$ and the white triangle the elliptic stagnation point of coordinates $(2.94,11.34)$.

krylov space is used to compute the leading adjoint eigenmodes as we did for the direct operator. Although the discretization of the continuous adjoint operator is different from the adjoint of the discrete direct operator, the resolution is large enough to retrieve the biorthogonality condition between the direct and adjoint eigenmodes (see Appendix A2, Eq. (A13)) with a very good accuracy. The spanwise vorticity, the enstrophy, and the energy of the adjoint mode of the leading eigenvalue are localised only on the contracting manifold of the hyperbolic stagnation point represented by a white star on Figs. 4(a)-4(c).

Fig. 5 shows the logarithm of the energy gain Eq. (9) with respect to time, for $\mathrm{k}_{\mathrm{z}}=3.4$ at $R_{e}=769$ and different initial conditions: the direct eigenmode $\mathbf{U}_{1}$ (thin line) and the adjoint eigenmode $\mathbf{U}_{1}^{+}$(thick line). For the direct mode, the gain in energy grows exponentially at a slope twice the growth rate computed using the Krylov Arnoldi technique: $\sigma_{r}=0.141$. The energy grows initially faster when initialized by the adjoint eigenmode and asymptotically reaches a straight line parallel to the direct mode case but with an extra gain in good agreement with the prediction of Eq. (A23) (Appendix A3): $1 /\left(\mathbf{U}_{1} \mid \mathbf{U}_{1}^{+}\right)^{2}=\exp (2.07)$, for $R_{e}=769$. The adjoint eigenmode is the optimal perturbation that maximizes the energy gain at large time (see the derivation for the linearized Navier-Stokes equations in Appendix).

It is quite remarkable that the adjoint eigenmode is dominated by the spanwise vorticity (Fig. 4(a)) with two opposite sign bands of vorticity, corresponding to an in-plane jet type perturbation, as visualized by the velocity field in the $(x, y)$ plane plotted on Fig. 6. Such jet type initial perturbation (Figs. 4(a) and 6(a)) generates at large time streamwise vortices since the direct mode presented on Figs. 3(g) and 3(h) is dominated by in-plane vorticity. Amazingly this scenario of streamwise jets generating streamwise vortices is the opposite of the lift-up mechanism active in shear flows, where streamwise vortices give rise to streamwise jets like perturbation called streaks. In order to get a better understanding of the physical mechanism of instability, we consider the linearized vorticity equation

$$
\frac{\partial \widetilde{\boldsymbol{\omega}}}{\partial t}=-\mathbf{U}_{B} \cdot \nabla \widetilde{\boldsymbol{\omega}}+\widetilde{\boldsymbol{\omega}} \cdot \nabla \mathbf{U}_{B}-\tilde{\mathbf{U}} \cdot \nabla \boldsymbol{\Omega}_{B}+\nabla \tilde{\mathbf{U}} \cdot \Omega_{B}+\frac{1}{R_{e}} \Delta \widetilde{\boldsymbol{\omega}},
$$

where the first two terms of the right hand side are, respectively, the transport and the stretching/tilting of the 


\section{$\mathrm{k}_{\mathrm{z}}=3.4$, OPTIMAL PERTURBATION at $\tau=\infty$}

(a)

Spanwise Vorticity

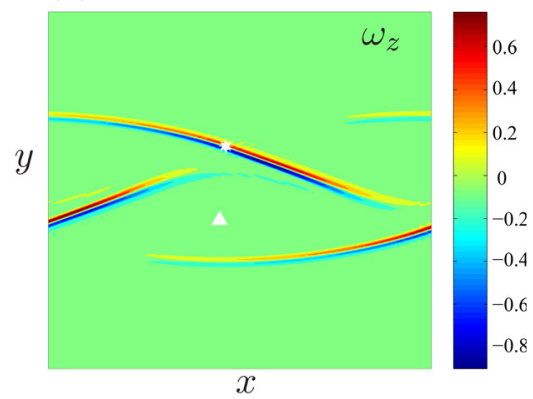

Enstrophy

(b)

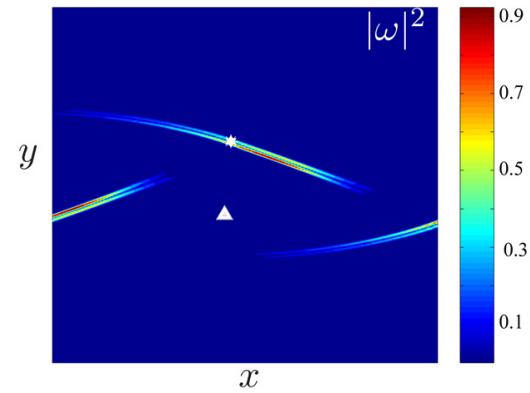

Kinetic Energy

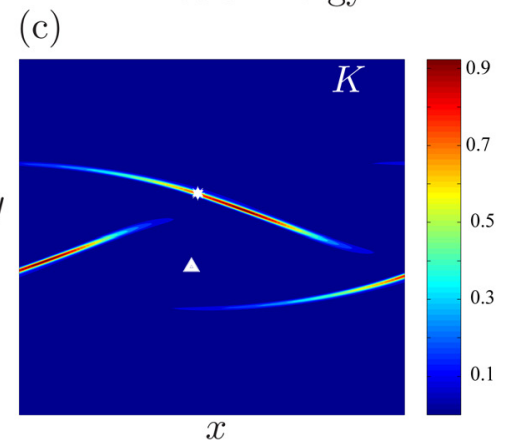

FIG. 4. (Color online) Symmetric mode: Spanwise vorticity field $\omega_{z}$ (a), enstrophy $|\omega|^{2}$ field (b), and kinetic energy K field (c) of the leading adjoint eigenmode in the $(\mathrm{x}, \mathrm{y})$ plane for $R_{e}=769$ and for $\mathrm{k}_{\mathrm{z}}=3.4$. The contour levels shown and the size of the domain are the same as Fig. 3. The white star locates the hyperbolic stagnation point which has coordinates $(3.04,14.38)$ and the white triangle, the elliptic stagnation point of coordinates $(2.94,11.34)$.

perturbation vorticity by the base flow and the following two terms, respectively, the transport and the stretching/tilting of the base flow vorticity by the perturbation. The vorticity $\widetilde{\boldsymbol{\omega}}$ and velocity $\tilde{\mathbf{U}}$ perturbations are decomposed on the form, $\widetilde{\boldsymbol{\omega}}=\widetilde{\boldsymbol{\omega}}_{p}+\widetilde{\boldsymbol{\omega}}_{z} \vec{e}_{z}$ and $\tilde{\mathbf{U}}=\tilde{\mathbf{U}}_{\mathbf{p}}+\tilde{u}_{z} \vec{e}_{z}$ with $\widetilde{\boldsymbol{\omega}}_{p}$ and $\tilde{\mathbf{U}}_{\mathbf{p}}$, respectively, the vorticity and velocity perturbations in the $(x, y)$ plane and $\widetilde{\omega}_{z}$ and $\tilde{u}_{z}$, respectively, the spanwise perturbed vorticity and velocity, $\vec{e}_{z}$ is the spanwise unit vector. As the in-plane base flow velocity $\mathbf{U}_{B}$ and the spanwise base flow vorticity $\boldsymbol{\Omega}_{\boldsymbol{B}}$ depend only on $(x, y)$ coordinates, the evolution equations for $\widetilde{\omega}_{p}$ becomes in the inviscid limit

$$
\frac{\partial \widetilde{\boldsymbol{\omega}}_{p}}{\partial t}+\mathbf{U}_{B} \cdot \nabla \widetilde{\boldsymbol{\omega}}_{p}=\frac{\partial \tilde{\mathbf{U}}_{p}}{\partial z} \cdot \Omega_{B}+\widetilde{\boldsymbol{\omega}}_{p} \cdot \nabla \mathbf{U}_{B} .
$$

All the vectors are, without introducing tedious notation, considered as two components vectors and gradients. The equation evolution for $\widetilde{\omega}_{z}$ arising from the projection of Eq. (13) on the spanwise direction evolves according to the equation

$$
\frac{\partial \widetilde{\omega}_{z}}{\partial t}+\mathbf{U}_{B} \cdot \nabla \widetilde{\omega}_{z}=-\tilde{\mathbf{U}}_{\mathbf{p}} \nabla \Omega_{B}+\frac{\partial \tilde{u}_{z}}{\partial z} \cdot \Omega_{B} .
$$

At finite and long time, the optimal initial perturbation consists of an in-plane jet type perturbation, the initial velocity is mainly in the $(x, y)$ plane $\left(\tilde{u}_{z} \sim 0\right)$ and is located on the contracting manifold of the hyperbolic point (Fig. 6), where the base flow vorticity is small but non zero (Fig. 1), $\tilde{\mathbf{U}}_{\mathbf{p}} \cdot \nabla \Omega_{B} \sim 0$; since the base flow vorticity is constant along trajectories in the inviscid limit. Hence initially, the spanwise vorticity $\tilde{\omega}_{z}$ Eq. (15) evolves at leading order according to the simple transport equation

$$
\frac{\partial \widetilde{\omega}_{z}}{\partial t}+\mathbf{U}_{B} \cdot \nabla \widetilde{\omega}_{z}=0
$$

Therefore, the spanwise perturbation vorticity evolves as a passive scalar convected by the base flow at the early time. The transient growth arises from the production of in-plane vorticity in the response which evolves according to Eq. (14). In Eq. 14, we found that the two terms, $\frac{\partial \tilde{\mathbf{U}}_{p}}{\partial z} \Omega_{B}=i \mathrm{k}_{\mathrm{z}} \tilde{\mathbf{U}}_{p} \Omega_{B}$ representing the tilting of the spanwise base flow vorticity by the alternated in $z$ in-plane jet, and $\widetilde{\boldsymbol{\omega}}_{p} . \nabla \mathbf{U}_{B}$, the stretching/tilting of the perturbation by the mean flow, initially lead the evolution eventhough $\Omega_{B}$ and $\widetilde{\omega}_{p}$ are small (Figs. 1(a) and 7(a)) since $\tilde{\mathbf{U}}_{p}$ and the strain are large on the contracting manifold of the hyperbolic point (Figs. 1(b) and 6(a)). At early time, the in-plane vorticity (white arrow on Fig. 7(a)), located along the contracting manifold of the hyperbolic point, is not exactly aligned with the stretching direction of the hyperbolic point (white dotted line on Fig. 7(a)). The tilting of the spanwise base flow vorticity by the in-plane jet, $\frac{\partial \tilde{\mathbf{U}}_{p}}{\partial z} \Omega_{B}$ in Eq. (14), tends to align the in-plane vorticity along the stretching manifold during its journey toward the hyperbolic point (Fig. 7(b)). At the same time, the in-plane vorticity is stretched by the mean flow $\widetilde{\boldsymbol{\omega}}_{p} . \nabla \mathbf{U}_{B}$ in Eq. (14). This stretching dominates later time.

(a)

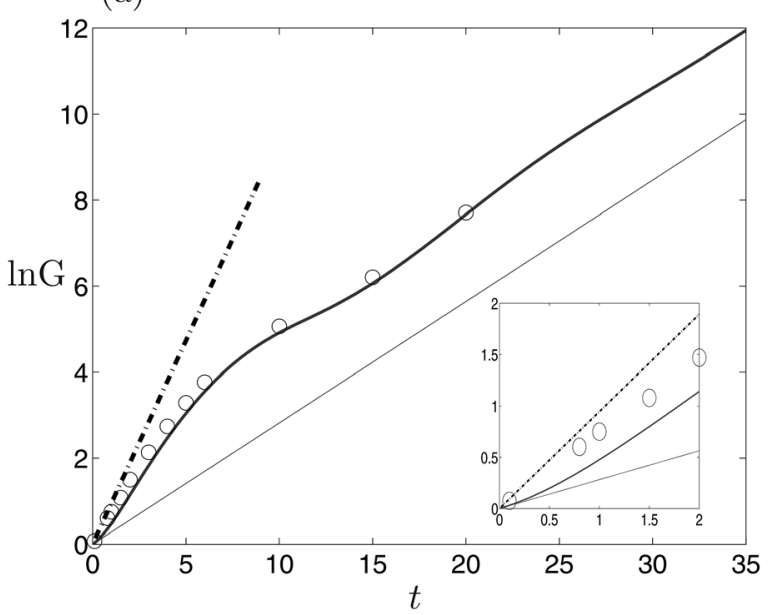

FIG. 5. Energy gain of symmetric mode for the most amplified spanwise wavenumber $\mathrm{k}_{\mathrm{z}}=3.4$ and for $R_{e}=769$ as a function of time. Thin continuous line: initial condition is the direct eigenmode presented Fig. 3. Heavy continuous line: initial condition is the adjoint eigenmode presented Fig. 4 optimal at large time. Open symbols: optimal gain at each time horizon: the initial condition is optimized and, therefore, different at each time horizon. Dashed-dotted line: theoretical prediction of the energy gain at short times Caulfield and Kerswell ${ }^{14}$ and Eq. (20). 
(a)

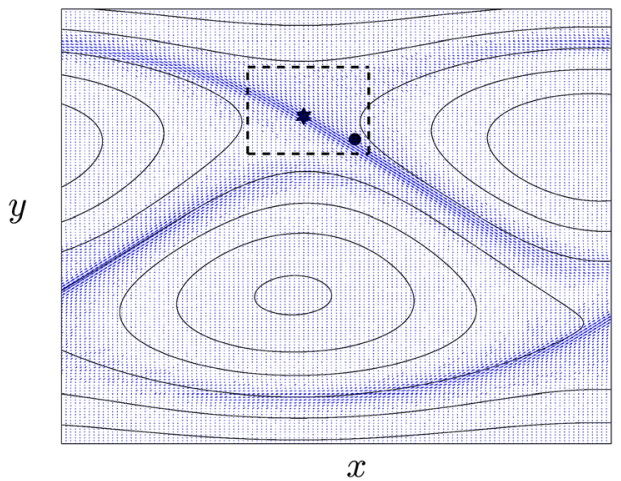

(b)

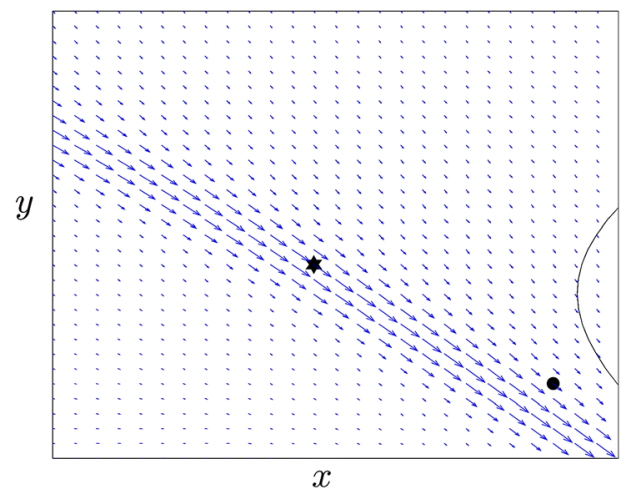

FIG. 6. (Color online) Velocity field of the leading adjoint eigenmode: The solid lines represent the streamlines of the basic flow. The arrows denote the velocity of the adjoint eigenmode in the $(\mathrm{x}, \mathrm{y})$ plane for $\mathrm{k}_{\mathrm{z}}=3.4$. The domain represented on (a) is $[0.49,2 \pi]$ in the $x$ direction and $[8.83,16.2]$ in the $y$ direction. The black star locates the hyperbolic stagnation point which has coordinates $(3.04,14.38)$, the "o" symbol is the point maximum of strain located at $(3.58,13.99)$. (b) Blow up of the area delimited by a rectangle on figure (a) around the hyperbolic point.
All along this evolution, the perturbation is transported by the base flow and eventually is localised along the stretching manifold of the hyperbolic point (Fig. 7(c)). Antkowiak and Brancher $^{19}$ observed in the case of a stable Lamb-Oseen gaussian vortex that optimal initial "streaks" of axisymmetric azimuthal velocity located at the periphery of the vortex give rise to amplified transient azimuthal vortices. They called this mechanism "anti lift-up" since the scenario was inverted compared to the classical lift-up mechanism in wake shear flow where streamwise vortices produce low and high speed streaks.

\section{B. Optimal perturbations}

We look for the optimal initial condition which maximizes the energy gain Eq. (9) at a finite time horizon $\tau$

$$
\mathrm{G}(\tau)=\max _{\tilde{U}(0)}\left(\frac{(\tilde{\mathbf{U}}(\tau) \mid \tilde{\mathbf{U}}(\tau))}{(\tilde{\mathbf{U}}(0) \mid \tilde{\mathbf{U}}(0))}\right) .
$$

As in Corbett and Bottaro, ${ }^{30}$ the optimal initial condition may be obtained by an iterative procedure, involving alternative integration of the direct and adjoint equations. The evolution of any initial condition, $\tilde{\mathbf{U}}(\mathbf{0})$, is obtained by the integration of Eq. (3) until the time $\tau$ leading to the velocity $\tilde{\mathbf{U}}(\tau)$. The result of this integration allows the formal definition of the propagator $\Phi(\tau)$

$$
\tilde{\mathbf{U}}(\tau)=\Phi(\tau) \tilde{\mathbf{U}}(0)
$$

Similarly, the backward integration of the adjoint linearized Navier Stokes equation (12) allows to define the adjoint propagator $\Phi^{+}(\tau)$, which turns out to be also the adjoint of $\Phi(\tau)$ with respect to the inner product Eq. (10). ${ }^{30}$ The optimal initial condition is then the eigenvector associated with the largest eigenvalue of the operator, $\Phi^{+}(\tau) \Phi(\tau)$, since the optimal gain is

$$
\mathrm{G}(\tau)=\max _{\tilde{U}(0)}\left(\frac{\left(\Phi^{+}(\tau) \Phi(\tau) \tilde{\mathbf{U}}(0) \mid \tilde{\mathbf{U}}(0)\right)}{(\tilde{\mathbf{U}}(0) \mid \tilde{\mathbf{U}}(0))}\right) .
$$

The operator $\Phi^{+}(\tau) \Phi(\tau)$ being hermitian positive definite is normal and the optimal perturbation corresponds then to its leading eigenmode, which may be simply computed via power iteration as done by Luchini. ${ }^{17}$ In practice, the direct equations are integrated until a time $t=\tau$, the backward integration in time of the adjoint equations is then performed using the final state of the direct integration as the initial perturbation for the adjoint equation. The procedure is reiterated until convergence defined in practice as when the absolute variation of $\ln \mathrm{G}$ is less than $10^{-2}$. The optimal energy gains at different instant are plotted on Fig. 5 as open circles, they are very close to the gain obtained when the initial condition is the adjoint eigenmode, suggesting that all the transient

EVOLUTION OF THE IN-PLANE ENSTROPHY $\left|\tilde{\omega}_{p}\right|^{2}$

(a)

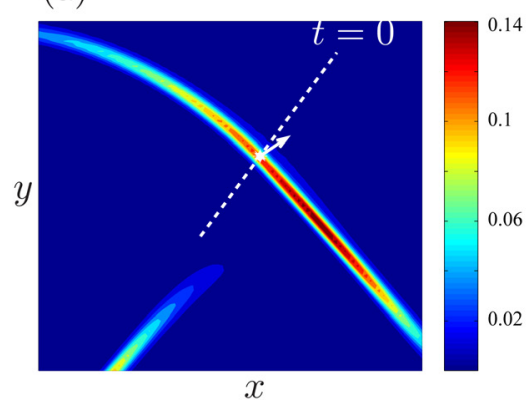

(b)

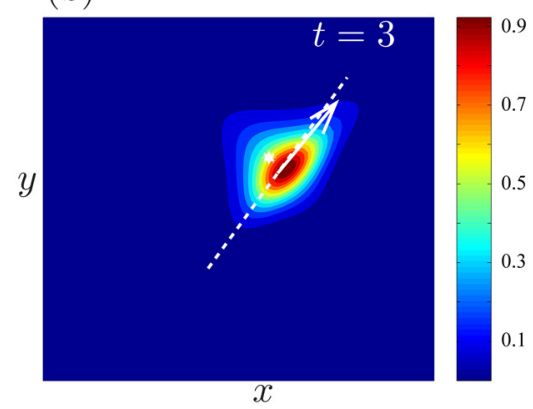

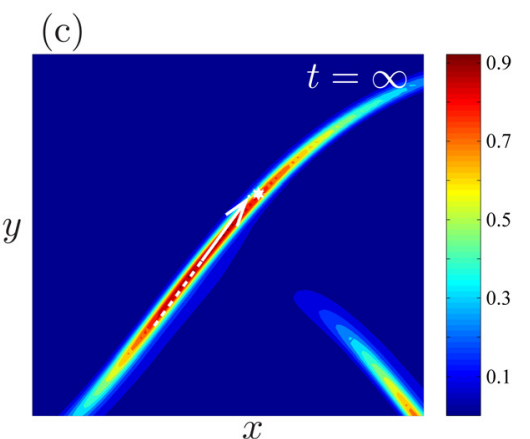

FIG. 7. (Color online) Evolution of the in-plane enstrophy $\left|\tilde{\omega}_{p}\right|^{2}$ of the in-plane jet perturbation (Fig. 6), for $\mathrm{k}_{\mathrm{z}}=3.4$ and $R_{e}=769$ : (a) optimal initial perturbation at $\mathrm{t}=0$, (b) optimal response for $\mathrm{t}=3$, and (c) evolution of the perturbation until $\tau=\infty$. Streamwise enstrophy has been normalized by the maximum of enstrophy. The domain represented is $[0.73,4.9]$ in the $\mathrm{x}$ direction and $[12.27,15.95]$ in the $y$ direction. The white arrows represent the in plane vorticity perturbation vector, the white dotted line the stretching direction and the hyperbolic point marked by a white star of coordinates $(3.04,14.38)$. 
dynamics is led by the adjoint mode. The transients last until $\tau \sim 20$, when the slope of the optimal gain curve $\ln (G)$ asymptotes to a $5 \%$ precision the one given by the leading instability growth rate.

\section{Finite time dynamics}

Fig. 8(e) presents the enstrophy of the optimal initial perturbation at time horizon $\tau=5$, Fig. 8(f) the corresponding optimal response both plotted in a zoomed area around the hyperbolic point. The optimal perturbation is initially centred on the point where the strain is maximum and is elongated along the contracting manifold of the hyperbolic point. Initially, $85 \%$ of its enstrophy is on the spanwise vorticity, whereas the response is dominated by in-plane vorticity $\widetilde{\omega}_{p}$ Eq. (14) which corresponds then to $99 \%$ of total enstrophy. This indicate that optimal response at time horizon $\tau=5$ produces already streamwise vortices via the "antiliftup" mechanism described above, the optimal initial perturbation being an plane jet as for the adjoint mode, the only difference being the streamwise extension of the perturbation, much more limited for $\tau=5$ than for $\tau=\infty$ (adjoint mode).

\section{Short time dynamics}

At very early time horizon, $\tau=0.1$, the optimal energy gain $\ln (G)=0.075$ is twice larger than the one obtained at the same time, when initializing by adjoint eigenmode $\ln (\mathrm{G})=0.032$. At this very short time, the optimal response Fig. 8(b) is nearly identical to the optimal perturbation Fig. 8(a) since the flow has no time to evolve. The initial optimal perturbation Fig. 8(a) and its correponding response Fig. 8(b) have, respectively, $79 \%$ and $83 \%$ of their enstrophy on the $(x, y)$ plane. The enstrophy of the optimal perturbation is concentrated close to the point of maximum strain, indicated by a white dot on Figs. 1 and 8 .

When time horizon is increased to $\tau=0.8$, the enstrophy of the optimal initial perturbation Fig. 8(c) keeps being concentrated near the point of maximum strain and the optimal response starts moving toward the hyperbolic point Fig. 8(d). At this time, the enstrophy of the optimal initial perturbation is due to in-plane vorticity perturbation $\tilde{\omega}_{p}$ which represents 98\% of the total enstrophy Fig. 9(a). Initially, the in-plane vorticity (white arrow on Fig. 9(a)) of the optimal perturbation is already aligned along the stretching direction of the hyperbolic point, the dominant term on Eq. (14) is mainly

\section{$\mathrm{k}_{\mathrm{z}}=3.4$, OPTIMAL PERTURBATION at finite time}

(a)

Optimal perturbation

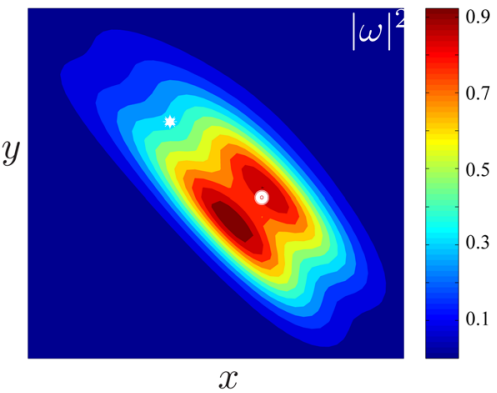

(c)

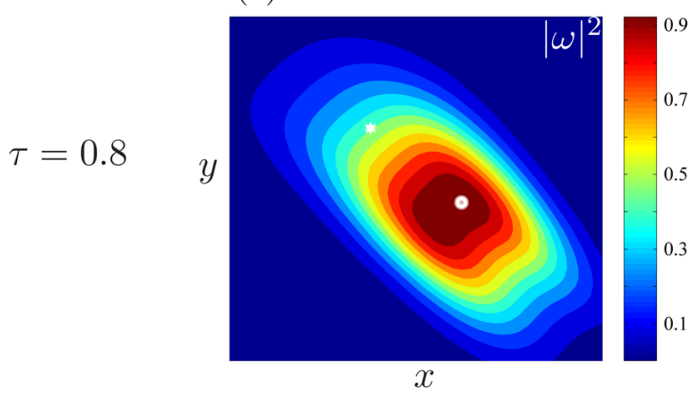

(e)

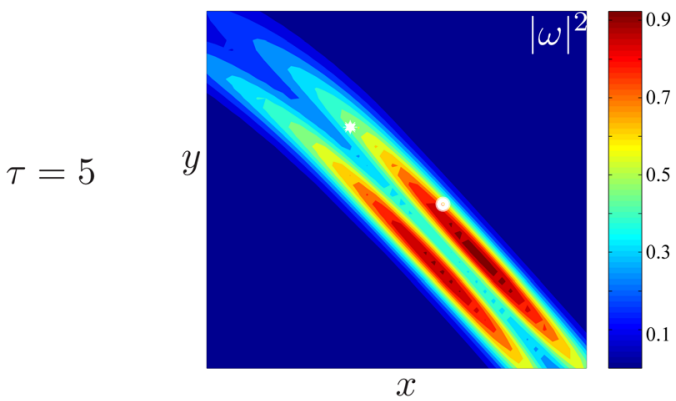

(b)

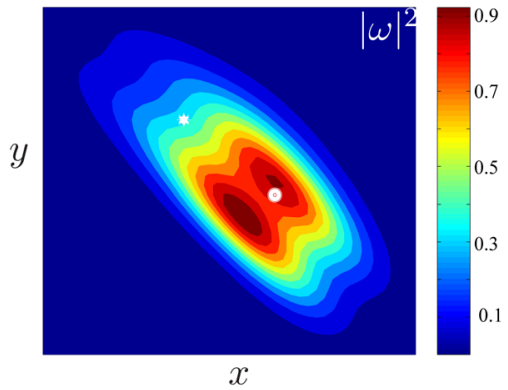

(d)

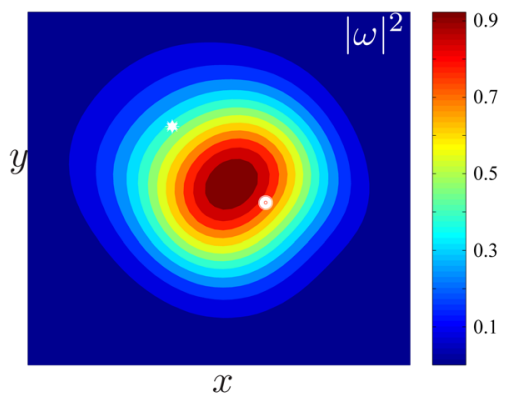

(f)

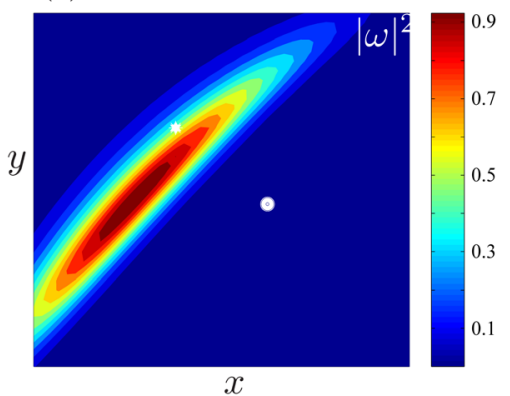

FIG. 8. (Color online) Optimal initial perturbations and corresponding response at different time horizons (a), (b) $\tau=0.1$, (c), (d) $\tau=0.8$, and (e), (f) $\tau=5$ : Close up view of the enstrophy field $|\omega|^{2}$ around the hyperbolic point for $R_{e}=769, \mathrm{k}_{\mathrm{z}}=3.4$ at time horizon: (a), (b) $\tau=0.1$, (c), (d) $\tau=0.8$, and (e), (f) $\tau=5$. The enstrophy is normalized by its maximum. The domain represented is $[2.26,4.51]$ in the $\mathrm{x}$ direction and $[13.06,14.98]$ in the $y$ direction. The white star locates the hyperbolic stagnation point of coordinates $(3.04,14.38)$ and the white point marked the location of the maximum of strain point of coordinates $(3.58,13.99)$ as on Fig. 1. 


\section{EVOLUTION OF THE IN-PLANE ENSTROPHY $\left|\tilde{\omega}_{p}\right|^{2}$}

(a)

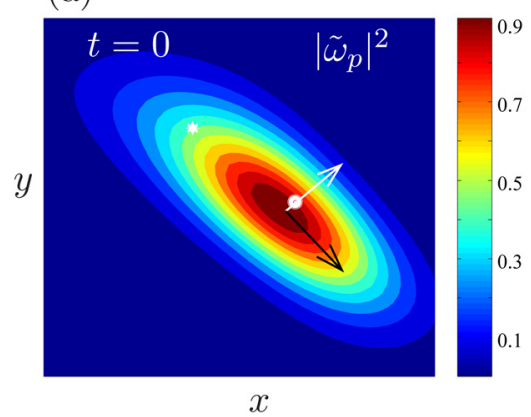

(b)

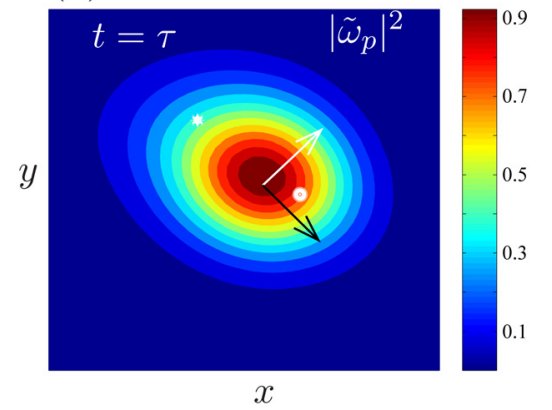

(c)

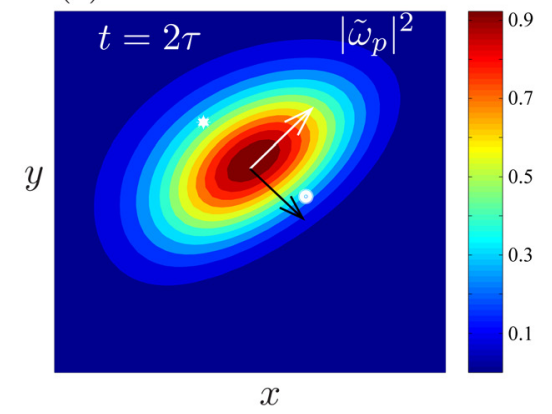

FIG. 9. (Color online) Evolution of the in-plane enstrophy $\left|\tilde{\omega}_{p}\right|^{2}$ of the optimal initial perturbation at time horizon $\tau=0.8$, for $\mathrm{k}_{\mathrm{z}}=3.4$ and $R_{e}=769$ : (a) optimal initial perturbation at $\mathrm{t}=0$, (b) optimal response for $\mathrm{t}=\tau$ (c) evolution of the perturbation until $\mathrm{t}=2 \tau$. Streamwise enstrophy has been normalized by the maximum of enstrophy. The black arrows represent the in-plane velocity and the white arrows the in plane vorticity perturbation vectors of the perturbation. The domain represented is $[2.26,4.51]$ in the $\mathrm{x}$ direction and $[13.06,14.98]$ in the $y$ direction. The white star locates the hyperbolic stagnation point of coordinates $(3.04,14.38)$ and the white point marked the location of the maximum of strain point of coordinates $(3.58,13.99)$.

the stretching of the perturbation by the base flow $\widetilde{\omega}_{p} . \nabla \mathbf{U}_{B}$. Caulfield and Kerswell ${ }^{14}$ have predicted in the case of an unbounded inviscid quadratic flows with a hyperbolic stagnation point in the framework of local theories (Refs. 12 and 32) that the optimal vorticity perturbation, leading to the maximum growth of energy at large time, should be aligned with the stretching direction of the hyperbolic point, the velocity perturbation of the corresponding response is in the $(x, y)$ plane, and along the contracting direction of the hyperbolic point. We plot on Fig. 9(a) by a white arrow the perturbed vorticity vector at the point of maximum perturbation amplitude and by a black arrow the perturbed velocity vector. The direction of both vectors is in excellent agreement whith local theory.

As shown by Corcos and Lin, ${ }^{31}$ the second term in Eq. (14), $\widetilde{\omega}_{p} \cdot \nabla \mathbf{U}_{B}$, enhances the streamwise vorticity with no change of the circulation. This mechanism linked to vortex stretching differs from the 2D Orr-mechanism for which the vorticity is in spanwise direction and is not affected by stretching. In the classical 2D Orr-mechanism in an unbounded constant shear flow, ${ }^{18}$ the velocity perturbation is on the form $\tilde{U}(t) \exp (i \mathbf{k}(t) \cdot \mathbf{x})$. The spanwise vorticity is conserved while the wave vector $\mathbf{k}(t)$, with no spanwise variation, rotates leading to transient growth of the in-plane velocity fields. In Caulfield and Kerswell, ${ }^{14}$ the wave vector is spanwise and does not evolve in time, and the in-plane vorticity is stretched and tilted by the mean flow $\left(\widetilde{\omega}_{p} . \nabla \mathbf{U}_{B}\right.$ in Eq. (14)) leading also to transient growth of the in-plane velocity. In the present case, the patch of vorticity initially centred on the point of maximum shear elongated along contracting manifold of the hyperbolic point adopt a rounded shape at $t=\tau$ Fig. 9(b) and starts being elongated along the stretching manifold at $t=2 \tau$ Fig. 9(c). At the same time, its centre is displaced toward the hyperbolic point. Such a localisation of the vorticity patch is not described in Caulfield and Kerswell $^{14}$ since their base flow is a uniform strain field extending to infinity.

Donnadieu et al $^{33}$ have shown, in the inviscid limit for the perturbation, that the initial condition which maximizes the energy gain Eq. (9) at short time is given by the eigenvector of the symmetric part of the base flow velocity gradient localised near the point of maximum strain. The corresponding eigenvalue is the maximum value of the base strain $\epsilon_{B}$ (Fig. 1(b))

$$
\ln \mathrm{G}(t)=-2 \max \left(\epsilon_{B}\right) t+O\left(t^{2}\right),
$$

a result which extends the results of Ref. 14 for an unbounded inviscid quadratic flow with an hyperbolic stagnation point, where the maximum of the growth rate is twice the strain rate $\epsilon$. This prediction at short time is represented on Fig. 5 by a dashed-dotted line and is in reasonable agreement with the computed optimal gain, considering the fact that Eq. (20) is an inviscid theory valid for large $k_{z}$, whereas presently the spanwise wavenumber and the Reynolds number are finite.

\section{DISCUSSION AND CONCLUSION}

\section{A. Effect of the wavenumber}

Up to now, the optimal gain has been presented only for the most unstable wavenumber $\mathrm{k}_{\mathrm{z}}=3.4$ but the procedure may be repeated for any $\mathrm{k}_{\mathrm{z}}$. For time horizon $\tau=5$ and above, we have found that whatever the wavenumber, the vorticity of the initial optimum perturbation is mainly in the spanwise direction corresponding to streamwise alternated jets concentrated on the contracting manifold of the hyperbolic point. The anti lift-up mechanism, therefore, leads the initial transient for all wavenumbers for time horizon larger than $\tau=5$. It should be noticed that for large time horizon $(\tau>20)$, the final response to this initial anti lift-up mechanism does depend on the wavenumber since it corresponds to the eigenmode plotted on Fig. 3 and discussed in Sec. II. For small wavenumbers $\left(\mathrm{k}_{\mathrm{z}} \leq 1\right)$, the kinetic energy of the response has a significant contribution in the core of the vortices (Fig. 3(c)) whereas the corresponding enstrophy is always localized on the stretching manifold of the hyperbolic point (Fig. 3(b)) and dominated by in-plane vorticity. For those wavenumbers another mechanism is present, transfering energy to the core by induction after time $\tau=20$. 
Only for short time horizon, the wavenumber has an effect and we restrict the discussion to time horizon $\tau=0.8$ and to three wavenumbers $\mathrm{k}_{\mathrm{z}}=1, \mathrm{k}_{\mathrm{z}}=2.4$, and $\mathrm{k}_{\mathrm{z}}=8$ on both sides of the instability peak (Fig. 2). The numerical calculations give an energy gain mostly independant of the wavenumber, $\ln G=0.55$ for $k_{z}=1$, $\ln G=0.59$ for $\mathrm{k}_{\mathrm{z}}=2.4$, ln $\mathrm{G}=0.6$ for $\mathrm{k}_{\mathrm{z}}=3.4$, and $\ln \mathrm{G}=0.56$ for $\mathrm{k}_{\mathrm{z}}=8$. The asymptotic theory (20) predicts also an energy gain independant of the wavenumber $\ln \mathrm{G}=0.76$, in good agreement with numerical calculations, considering the fact that Eq. (20) is obtained in the inviscid limit. We plot on Figs. 10(a), $10(\mathrm{c})$, and $10(\mathrm{e})$ the in-plane enstrophy $\left|\tilde{\omega}_{p}\right|^{2}$ of the optimal initial perturbations, respectively, for $\mathrm{k}_{\mathrm{z}}=1, \mathrm{k}_{\mathrm{z}}=2.4$, and $\mathrm{k}_{\mathrm{z}}=8$ and their corresponding response at $\tau=0.8$ on Figs. $10(\mathrm{~b}), 10(\mathrm{~d})$, and $10(\mathrm{f})$. At small wavenumber, $\mathrm{k}_{\mathrm{z}}=1$, the optimal initial perturbation and its corresponding response have, respectively, $8 \%$ and $17 \%$ of their enstrophy in the $(x, y)$ plane Figs. 10(a) and 10(b). As the wavenumber increases, the part of the in-plane enstrophy increases. Optimal initial perturbation have, respectively, $64 \%$ for $\mathrm{k}_{\mathrm{z}}=2.4$ and $98 \%$ for $\mathrm{k}_{\mathrm{z}}=8$ Fig. 10(c) and Fig. 10(e) of their enstrophy on the $(x, y)$ plane, and the in-plane enstrophy of their corresponding response are, respectively, $91 \%$ and $99 \%$ of the total enstrophy. For wavenumbers $\mathrm{k}_{\mathrm{z}}=2.4, \mathrm{k}_{\mathrm{z}}=3.4$, and $\mathrm{k}_{\mathrm{z}}=8$ transient growth of energy is mainly due, as described in Sec. III B 2, to the in-plane vorticity stretching similar to the hyperbolic instability. ${ }^{14}$ Only for small wavenumber $\mathrm{k}_{\mathrm{z}}=1$ the mechanism differs, the spanwise vorticity of the optimal initial perturbation is the dominant component and produces inplane enstrophy responsible for the transient growth; the part of in-plane enstrophy in the total enstrophy increases from $8 \%$ and $17 \%$ as time increases from $t=0$ to the time horizon $\tau=0.8$.

\section{B. Effect of the Reynolds number on the base flow}

As pointed out by Lin and $\mathrm{Corcos}^{20}$ and Neu, ${ }^{21}$ the secondary vortices which develop on stretched shear layers in the braid region are the result of a balance between the strain and the viscous diffusion. High-resolution simulations with lower kinematic viscosity have been performed to examine the effect of an higher Reynolds number on both the base flow and on the three dimensional perturbations.

Fig. 11 shows the base flow vorticity and the base strain for a larger Reynolds number $R_{e}=769$ with $256 \times 1024$ collocation points computed with the procedure described in Sec. II. For this Reynolds number, isovorticity lines match closely with streamlines (dark lines on Fig. 11(a)). The

\section{OPTIMAL PERTURBATION at $\tau=0.8$}

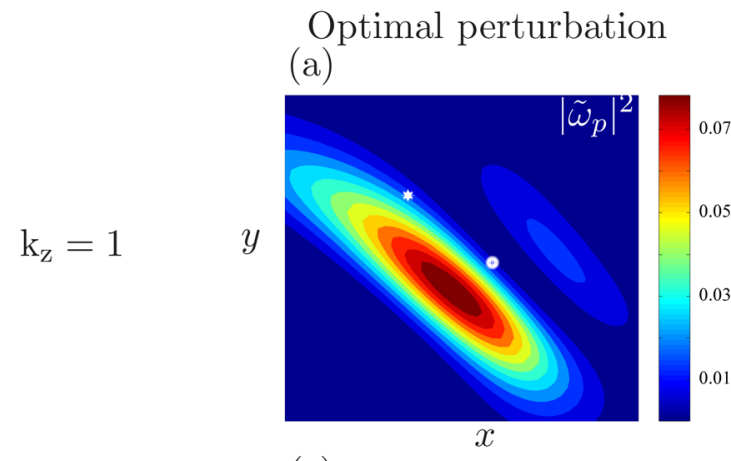

(c)

$\mathrm{k}_{\mathrm{z}}=2.4$

$y$

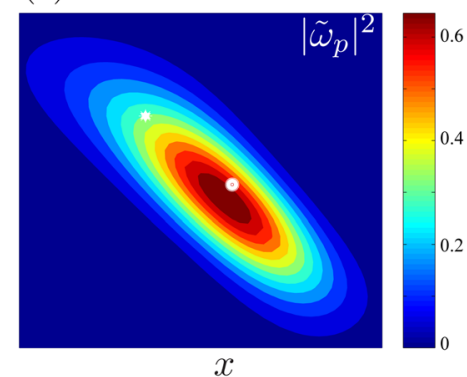

(e)

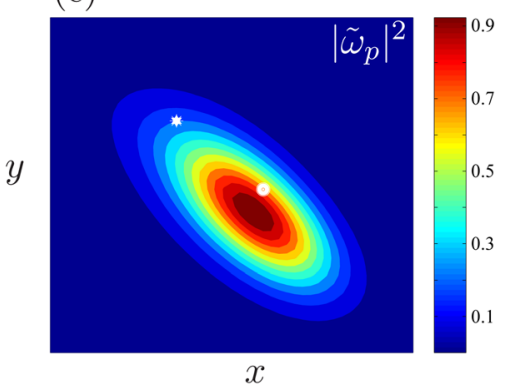

$$
\mathrm{k}_{\mathrm{z}}=8
$$

(b)

Optimal response

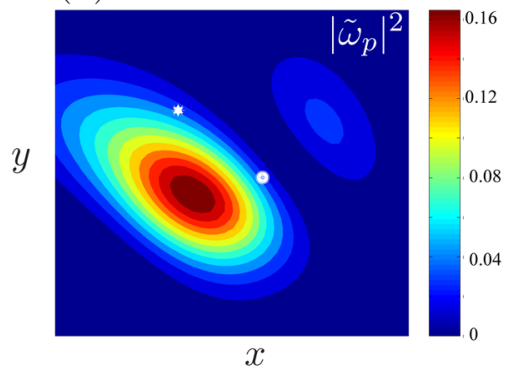

(d)

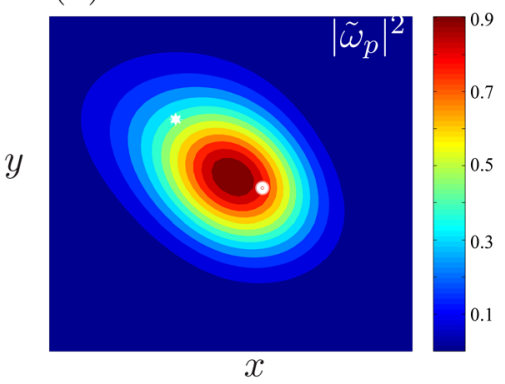

(f)

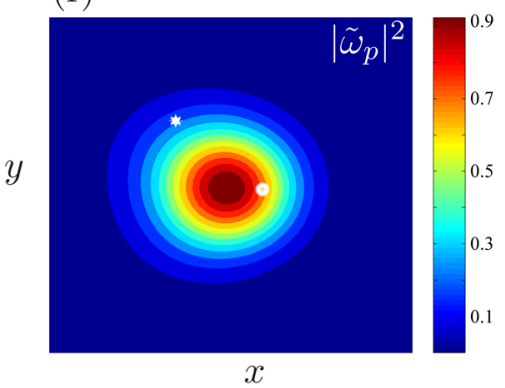

FIG. 10. (Color online) Optimal initial perturbations and corresponding response (b): Close up view of the inplane enstrophy field $\left|\tilde{\omega}_{p}\right|^{2}$ around the hyperbolic point for $R_{e}=769$ at time horizon $\tau=0.8$ for (a), (b) $\mathrm{k}_{\mathrm{z}}=1$, (c), (d) $\mathrm{k}_{\mathrm{z}}=2.4$, and (e), (f) $\mathrm{k}_{\mathrm{z}}=8$. The inplane enstrophy is normalized by the maximum of the total enstrophy $|\omega|^{2}$. The domain represented is [2.26, 4.51] in the $\mathrm{x}$ direction and $[13.06,14.98]$ in the y direction. The white star locates the hyperbolic stagnation point of coordinates $(3.04,14.38)$ and the white point marked the location of the maximum of strain point of coordinates $(3.58,13.99)$. 
(a)

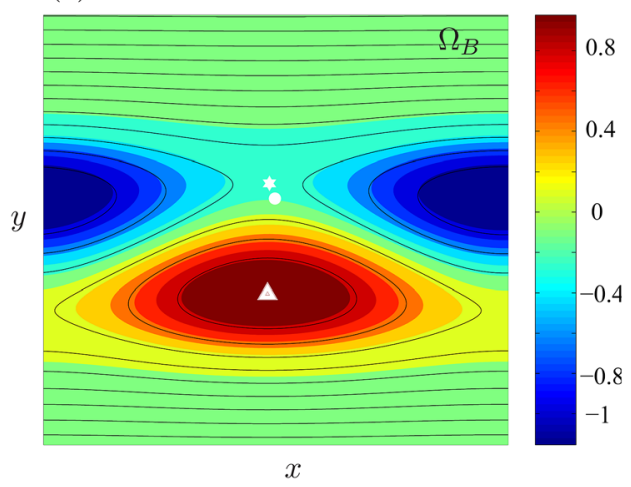

(b)

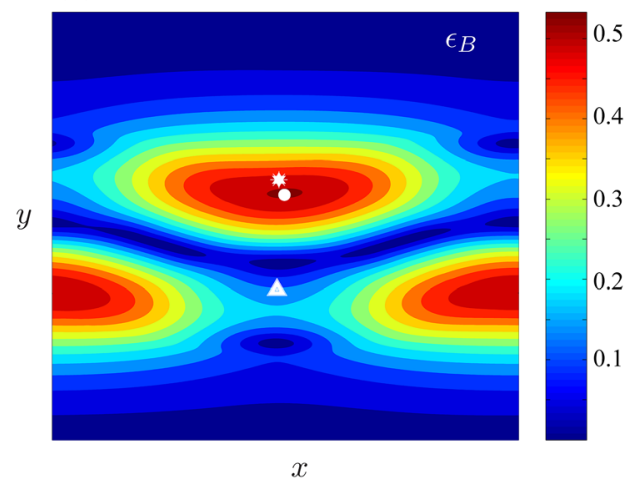

FIG. 11. (Color online) Same as Fig. 1 but for $R_{e}=769$ and $256 \times 1024$ collocation points. maximum of strain is $\epsilon_{B}=0.53$ and the strain at the hyperbolic point is $\epsilon_{B}=0.50$. The evolution of the base flow due to viscous diffusion is extremely slow and as explained in Sec. II, a temporal linear stability analysis is performed on this frozen base state Fig. 11.

For the base flow of Fig. 11, the evolution of the logarithm of the energy gain for the three-dimensional symmetric mode at $R_{e}=769$ with $\mathrm{k}_{z}=3.4$ is represented on Fig. 12 when the initial condition is the adjoint eigenmode (heavy dotted line) and with the direct eigenmode as the initial condition (thin dotted line). At large time, the slope of $\ln \mathrm{G}$ is twice the growth rate of three dimensional perturbation, for $\mathrm{k}_{z}=3.4$ and $R_{e}=769$, its value is $\sigma_{r}=0.138$. The logarithm of the energy gain is extremely close to the one obtained at the same wavenumber and same Reynolds number $R_{e}=769$ but for a perturbation superposed to the base state computed for a different Reynolds number $R_{e}=76.9$ (Fig. 1) and represented on Fig. 12 by continuous line (heavy lines: initialization by the adjoint eigenmode and light line: initialization by the direct eigenmode) showing that the value of the Reynolds number at which the base state has been computed does not affect the growth rate of the secondary perturbation on which

\section{INFLUENCE OF THE BASE FLOW}

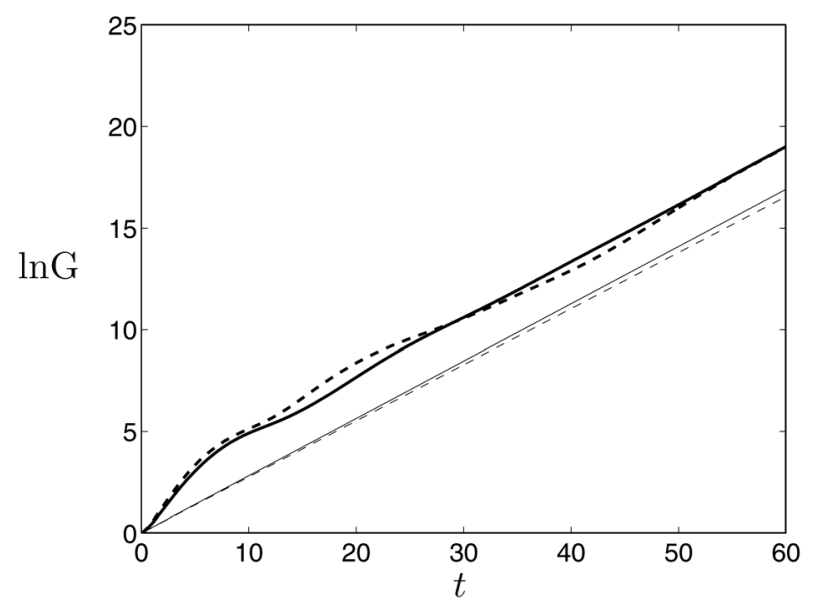

FIG. 12. Logarithm of the energy gain for the most amplified mode at $R_{e}=769$ and $\mathrm{k}_{\mathrm{z}}=3.4$ : Continuous lines: the perturbation is superposed to the base state computed at $R_{e}=76.9$ (Fig. 1). Dotted lines: the perturbation is superposed to the base state computed at $R_{e}=76911$. Heavy lines: the initial condition is the adjoint eigenmode. Light lines: the initial condition is the direct eigenmode. weaker dissipation may be applied (different Reynolds number for the perturbation). The extra gain $\ln \left(1 /\left(\mathbf{U}_{1} \mid \mathbf{U}_{1}^{+}\right)^{2}\right)$ (Appendix A3. Eq. (A23)) obtained when the adjoint perturbation is used as an initial condition is also mainly independent of the base flow chosen (Fig. 12). Thus, for a given Reynolds number for the perturbation dissipation, the non normality of three-dimensional perturbations are also mostly independant on the Reynolds number at which the base flow has been computed.

\section{Effect of the Reynolds number on the perturbations}

For the same base flow shown on Fig. 11 and for several Reynolds numbers $R_{e}=76.9, R_{e}=769, R_{e}=3333$, and $R_{e}=10000$, we have computed the wavenumber that maximizes the temporal growthrate, respectively, $\mathrm{k}_{\mathrm{z}}=1.2$, $\mathrm{k}_{\mathrm{z}}=3.4, \mathrm{k}_{\mathrm{z}}=5.4$, and $\mathrm{k}_{\mathrm{z}}=6.4$. For these wavenumbers, we have computed the adjoint eigenmode and measured at the hyperbolic point the thickness $\Delta$ of the energy containing region of the adjoint eigenmode and the thickness $\delta$ of the enstrophy of the direct eigenmode. The thickness $\Delta$ is defined as the distance beyond which the energy of the adjoint eigenmode drops below $10 \%$ of its maximum and the thickness $\delta$ is the same for the enstrophy of the direct eigenmode. The logarithm of these lengths is plotted on Fig. 13(a) with respect to the logarithm of the Reynolds number. The thickness $\delta$ (' $\star^{\prime}$ symbols) of the direct mode scales as $1 / \sqrt{R_{e}}$ in agreement with Lin and $\operatorname{Corcos}^{20}$ and Neu. ${ }^{21}$ The thickness $\Delta$ (' $\Delta^{\prime}$ symbols) of the adjoint energy obeys the same scaling. As a result, the localisation of the adjoint eigenmode on the contracting manifold of the hyperbolic point and of the direct eigenmode of the stretching manifold of the hyperbolic point is more pronounced when the Reynolds number increases.

As discussed on Figs. 5 and 12 and Appendix A3 (Eq. (A23), $\ln \left(1 /\left(\mathbf{U}_{1} \mid \mathbf{U}_{1}^{+}\right)^{2}\right)$ represents the extra gain obtained by initializing the perturbation by the adjoint eigenmode and it measures the non normality of the leading mode. The extra gain has been computed for the present set of Reynolds numbers and associated leading spanwise wavenumber. As shown of Fig. 13(b), the extra gain increases slowly with the Reynolds number. The reason for the increase of the non normality with the Reynolds number is linked to the fact that the adjoint eigenmode and the direct eigenmode are localised on different regions of the flow, as a result the product of the 
(a)

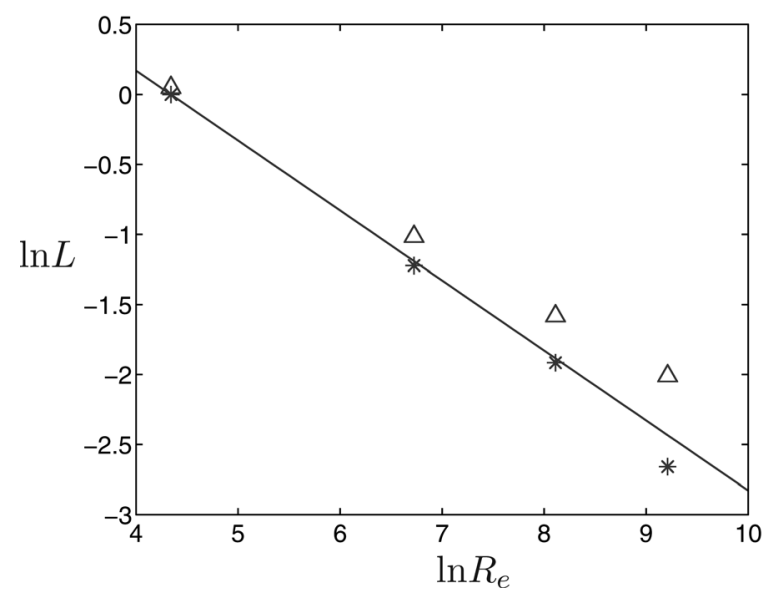

(b)

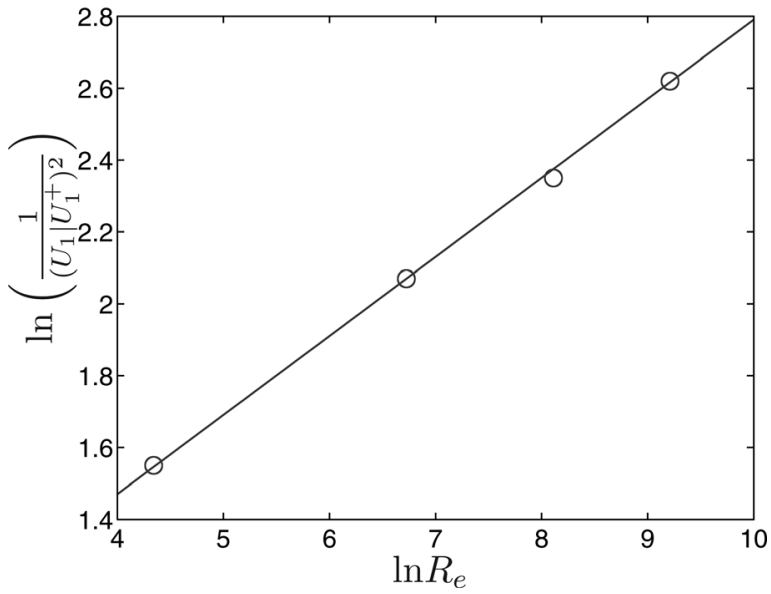

FIG. 13. Influence of the Reynolds number: (a) ' $\star$ ' symbol, Logarithm of the thickness of the enstrophy of direct eigenmode of the most amplified spanwise wavenumber measured at the hyperbolic point, ' $\Delta$ 'symbol, thickness of the energy of the adjoint eigenmode of the most amplified spanwise wavenumber measured at the hyperbolic point with respect to the logarithm of the Reynolds number. (b) Extra gain of energy for the most amplified wavenumber with respect to the logarithm of the Reynolds number. Perturbations have been superposed to the base flow shown on Fig. 11.

amplitude of their velocities (square of their kinetic energy) is almost zero everywhere (Fig. 14(a)), except in two regions, one around the hyperbolic point and the second on the contracting manifold. As shown on Fig. 14(b), the local scalar product of the velocities of the adjoint and direct eigenmodes $\mathbf{U}_{1} \cdot \mathbf{U}_{1}^{+}$is different from the product of their amplitudes Fig. 14(a) since concentrated in a single region around the hyperbolic point. The larger the Reynolds number, the more concentrated is the local scalar product $\mathbf{U}_{1} \cdot \mathbf{U}_{1}^{+}$ explaining the increase of the non normality shown on Fig. 13(b).

The quasi-static stability analysis of this paper is valid as long as the finite dimensional time horizon $\tau^{\star}=\left(\tau\left(\delta_{w} / \delta\right)\right) /(\bar{U} / 2)$ is smaller than the diffusion time scale of the base flow $\left.\tau_{B}=\left(\delta_{w} / \delta\right)\right)^{2} / \nu$, which implies $\tau^{\star} \ll \tau_{B}$ i.e., $\tau \ll R_{e}$. This condition is fulfilled for all the transient regimes investigated for the base flow of Fig. $11\left(R_{e}=769\right)$ and for short and finite horizon time for the base flow of Fig. $1\left(R_{e}=76.9\right)$, at larger time of optimization $(\tau>20)$ for this last case $\left(R_{e}=76.9\right)$, this condition is not well satisfied and optimization should be carried out letting the base flow diffuse since the direct-adjoint technique allows for unsteady base flow. However, the use of unsteady base flow would add the complexity of a competition between the primary instability development and the growth of secondary perturbation, out of the scope of the present paper which focused on the identification of different secondary instability mechanism and in particular the anti lift-up mechanism active after the roll up completed. These mechanisms and in particular the anti lift-up mechanism are generic, since they are independant both of the Reynolds number at which the base flow is computed and of the Reynolds number used for the linearized equation.

\section{CONCLUSION}

Results discussed above for $\mathrm{k}_{\mathrm{z}}=3.4$ and extended for all wavenumber show that the transient growth is triggered by different components of the vorticity perturbation at short and long time. At very short time $\tau=0.1$ and $\tau=0.8$, the dynamics of the wake are well predicted by the local instability theory of the hyperbolic instability ${ }^{14}$ both for the growth rate Eq. (20) and the localisation and orientation of optimum vorticity perturbation of initial optimal perturbation and its response. Only for short wavelength, the mechanism differs from the hyperbolic instability. At larger time of optimization, the mechanism is different, involving initially the anti lift-up, reminiscent of the one described by Antkowiak and (a)

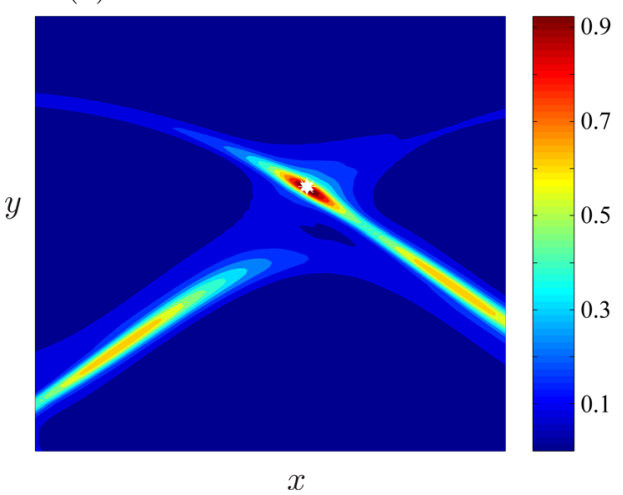

(b)

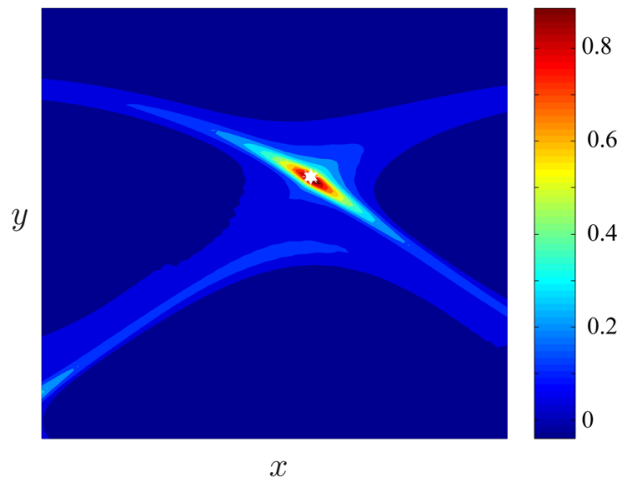

FIG. 14. (Color online) (a) Field of the product of the amplitude of the velocity of the direct and adjoint leading eigenmodes for $R_{e}=769$ and $\mathrm{k}_{\mathrm{z}}=3.4$ and the base flow plotted on Fig. 1: $\left|\mathbf{U}_{1}\right| .\left|\mathbf{U}_{1}^{+}\right|$ field normalized by its maximum. (b) Scalar product field $\mathbf{U}_{1} \cdot \mathbf{U}_{1}^{+}$, normalized by the maximum of $\left|\mathbf{U}_{1}\right| \cdot\left|\mathbf{U}_{1}^{+}\right|$. The domain represented on (a) and (b) is [2.73, $4.9]$ in the $x$ direction and $[10.55,16.69]$ in the y direction. The white star locates the hyperbolic stagnation point of coordinates $(3.04,14.38)$. 
Brancher ${ }^{19}$ where the production is associated with the stretching/tilting of the radial vorticity perturbation, associated with the azimuthal velocity perturbation located in the periphery of the vortex (quasi-potential region), by the base flow, for which the azimuthal velocity varies with the radial coordinate, leading to vortex rings formation around the vortex core. Here, the in-plane jet initial perturbation located along the contracting manifold of the hyperbolic point is tilting the base flow vorticity and at the same time, the in-plane vorticity perturbation is stretched by the base flow (as in Ref. 19). The non normality measured here by the scalar product of the direct and adjoint eigenmodes i.e., the integral over the domain of the field presented on Fig. 14(b), can be then attributed to two physical mechanisms that add up: the convective non normality $^{22}$ which is the result of the transport of the perturbation by the base flow and changes sign between direct (A1) and adjoint (A4) equations resulting in a different localisation of the receptivity (adjoint eigenmode) and response (direct eigenmode) and a componentwise non normality, due to the anti lift-up mechanism, in-plane jets (adjoint eigenmode) producing streamwise vortices (direct eigenmode). This later effect concentrates direct and adjoint modes on different components, explaining why the local scalar product $\mathbf{U}_{1} \cdot \mathbf{U}_{1}^{+}$vanishes away from the hyperbolic point in region where $\left|\mathbf{U}_{1}\right| \cdot\left|\mathbf{U}_{1}^{+}\right|$is not zero.

\section{APPENDIX: OPTIMAL PERTURBATION AT LARGE TIME, THE ADJOINT EIGENMODE}

The reader is referred to the work of $\mathrm{Hill}^{29}$ for the derivation of the adjoint Eq. (12) of the linearized Navier Stokes Equations (3), a brief review is given in Appendix A1. We will derive a relation between adjoint and direct solutions in order to prove that the adjoint mode provides the initial condition that maximizes the energy amplification factor (9) at large time. Farrell ${ }^{34}$ for a plane parallel shear flow, and for two dimensional instabilities governed by the OrrSommerfeld equation, has demonstrated that the adjoint mode is the optimal initial condition in the L2 norm. We generalized this result in the case of the linearized Navier-Stokes equations and an arbitrary 2D base flow and 3D perturbation.

\section{Continuity equation}

The direct linearized Navier-Stokes Equations (3) are rewritten in the form

$$
\begin{aligned}
\mathcal{L} \mathbf{f} & =\left(\begin{array}{cc}
\frac{\partial \tilde{\mathbf{U}}}{\partial t}+\Omega_{\mathbf{B}} \times \tilde{\mathbf{U}}+\widetilde{\boldsymbol{\omega}} \times \mathbf{U}_{\mathbf{B}}+\nabla\left[\widetilde{\mathbf{U}} \cdot \mathbf{U}_{\mathbf{B}}\right]+\nabla[\tilde{p}]-\frac{1}{R_{e}} \Delta \tilde{\mathbf{U}} \\
\nabla \cdot \tilde{\mathbf{U}}
\end{array}\right) \\
& =0,
\end{aligned}
$$

with $\mathbf{f}=(\tilde{\mathbf{U}}, \tilde{p})$ the direct state vector. The classical Lagrange identity (Ince ${ }^{35}$ ) is used to construct the linearized adjoint Navier-Stokes equation $\mathcal{L}^{+}$

$$
(\mathcal{L} \mathbf{f})^{* T} \mathbf{f}^{+}-\mathbf{f}^{* T}\left(\mathcal{L}^{+} \mathbf{f}^{+}\right)=\frac{\partial\left(\tilde{\mathbf{U}} \cdot \tilde{\mathbf{U}}^{+}\right)}{\partial t}+\nabla . \mathbf{J}\left(\tilde{\mathbf{U}}, \tilde{\mathbf{U}}^{+}, \tilde{p}, \tilde{p}^{+}\right),
$$

with the superscripts ${ }^{*}$ and ${ }^{T}$ denoting the complex conjugate and the transposition. The adjoint state vector is $\mathbf{f}^{+}$ $=\left(\tilde{\mathbf{U}}^{+}, \tilde{p}^{+}\right)$and the components $\mathbf{J}_{j}$ of the vector $\mathbf{J}$, called the bilinear concomitant $\left(\mathrm{Hill}^{29}\right)$, are

$$
\mathbf{J}_{j}=\tilde{\mathbf{U}}_{i}^{+}\left(\tilde{p} \delta_{i j}-\frac{1}{R_{e}} \frac{\partial \tilde{\mathbf{U}}_{i}}{\partial x_{j}}+U_{B_{j}} \tilde{\mathbf{U}}_{i}\right)+\left(\tilde{p}^{+} \delta_{i j}+\frac{1}{R_{e}} \frac{\partial \tilde{\mathbf{U}}_{i}^{+}}{\partial x_{j}}\right) \tilde{\mathbf{U}}_{i},
$$

(note that $\left(x_{1}, x_{2}, x_{3}\right)$ correspond to $\left.(x, y, z)\right)$ and $\mathcal{L}^{+}$

$$
\begin{aligned}
\mathcal{L}^{+} \mathbf{f}^{+} & =\left(\begin{array}{c}
\nabla \cdot \tilde{\mathbf{U}}^{+} \\
-\frac{\partial \tilde{\mathbf{U}}^{+}}{\partial t}-\Omega_{\mathbf{B}} \times \tilde{\mathbf{U}}^{+}+\nabla \times\left(\mathbf{U}_{\mathbf{B}} \times \tilde{\mathbf{U}}^{+}\right)+\nabla\left[\tilde{p}^{+}\right]-\frac{1}{R_{e}} \Delta \tilde{\mathbf{U}}^{+}
\end{array}\right) \\
& =0 .
\end{aligned}
$$

The two operators, $\mathcal{L}$ and $\mathcal{L}^{+}$, are adjoint for the inner product defined in Eq. (11), if the right hand side of Eq. (A2) vanishes when integrating over the box of numerical integration and over time

$$
\begin{gathered}
\int_{0}^{\tau} \int_{0}^{L x} \int_{0}^{L y} \frac{\partial\left(\tilde{\mathbf{U}} \cdot \tilde{\mathbf{U}}^{+}\right)}{\partial t} \mathrm{dxdydt}+\int_{0}^{\tau} \int_{0}^{L y} \int_{0}^{L x} \frac{\partial J_{z}}{\partial z} \mathrm{dxdydt} \\
\quad+\int_{0}^{\tau} \int_{0}^{L y}\left[J_{x}\right]_{0}^{L_{x}} \mathrm{dydt}+\int_{0}^{\tau} \int_{0}^{L x}\left[J_{y}\right]_{0}^{L_{y}} \mathrm{dydt}=0,
\end{gathered}
$$

with $J_{x}, J_{y}$, and $J_{z}$ the components of $\mathbf{J}$ (A3). The boundary conditions of the direct problem are periodic on the box of integration. For Eq. (A5) to be zero, we must apply periodic conditions in space to the adjoint problem and an initial condition in time, which is the final integration of the direct problem.

Two solutions $\mathbf{f}$ and $\mathbf{f}^{+}$of Eqs. (A1) and (A4) satisfy a continuity equation (the right hand member of Eq. (A2) is zero) as also shown by Salwen ${ }^{36}$

$$
\frac{\partial\left(\tilde{\mathbf{U}} \cdot \tilde{\mathbf{U}}^{+}\right)}{\partial t}+\nabla \cdot \mathbf{J}\left(\tilde{\mathbf{U}}, \tilde{\mathbf{U}}^{+}, p, p^{+}\right)=0 .
$$

The integration over space of the continuity Eq. (A6) gives the following relation:

$$
\frac{\partial}{\partial t}\left(\tilde{\mathbf{U}} \mid \tilde{\mathbf{U}}^{+}\right)+\frac{\partial}{\partial z}\left[\mathbf{f} \mid \mathbf{f}^{+}\right]=0
$$

with (I) the energy product (10) and

$$
\left[\mathbf{f} \mid \mathbf{f}^{+}\right]=\int_{0}^{L x} \int_{0}^{L y} J_{z} \mathrm{dxdy}
$$

note that [.I.] is not positive definite.

This equation was first introduced by Salwen ${ }^{36}$ and Salwen and $\mathrm{Grosh}^{37}$ to derive the biorthogonality relation for both temporal and spatial direct and adjoint eigenmodes of the Orr-Sommerfeld equation.

\section{Biorthogonality condition}

The direct and adjoint eigenfunctions are taken under the form of normal modes 


$$
\mathbf{f}_{\mathrm{kn}}=\left[\mathbf{U}_{\mathrm{kn}}, p_{\mathrm{kn}}\right](\mathrm{x}, \mathrm{y}) \mathrm{e}^{\mathrm{ikz}} \mathrm{e}^{\sigma_{k n} t}
$$

and

$$
\mathbf{f}_{\mathrm{k}^{+} \mathrm{n}}^{+}=\left[\mathbf{U}_{\mathrm{k}^{+} \mathrm{m}}^{+}, p_{\mathrm{k}^{+} \mathrm{m}}\right](\mathrm{x}, \mathrm{y}) \mathrm{e}^{\mathrm{ik}^{+} \mathrm{z}} \mathrm{e}^{\sigma_{\mathrm{k}^{+} \mathrm{m}}^{+}} .
$$

To a given transverse wavenumber $\mathrm{k}$ (resp. $\mathrm{k}^{+}$) corresponds a set of discrete modes of complex frequency $\sigma_{\mathrm{kn}}$ (respectively, $\sigma_{\mathrm{k}^{+} \mathrm{m}}^{+}$) with $\mathrm{n}$ (respectively, $\mathrm{m}$ ) denoting a particular mode.

As $\mathbf{f}_{\mathrm{kn}}$ and $\mathbf{f}_{\mathrm{kn}}^{+}$are solutions of direct Eq. (A1) and adjoint Eq. (A4) linearized Navier-Stokes equations. They satisfy the continuity relation (A7)

$$
\begin{aligned}
& e^{\left(-i \mathbf{k}^{\star}+i \mathrm{k}^{+}\right) z+\left(\sigma_{k n}^{\star}+\sigma_{k^{+} m}^{+}\right) t}\left(\left(\sigma_{k n}^{\star}+\sigma_{k^{+} m}^{+}\right)\left(\mathbf{U}_{k n} \mid \mathbf{U}_{k^{+} m}^{+}\right)\right. \\
& \left.+\left(-\mathrm{k}^{\star}+\mathrm{k}^{+}\right)\left[\mathbf{f}_{k n} \mid \mathbf{f}_{k^{+} m}^{+}\right]\right)=0 .
\end{aligned}
$$

In the case of a temporal study, $\mathrm{k}$ is real and we look for the adjoint mode corresponding to the same transverse wavenumber $\mathrm{k}^{+}=\mathrm{k}$. Therefore, $-\mathrm{k}^{\star}+\mathrm{k}^{+}=0$, and we obtain the following relation:

$$
\left(\sigma_{\mathrm{kn}}^{\star}+\sigma_{\mathrm{km}}^{+}\right)\left(\mathbf{U}_{\mathrm{kn}} \mid \mathbf{U}_{\mathrm{km}}^{+}\right)=0 .
$$

Hence, we retrieve in the three dimensional case, the biorthogonal condition derived by $\mathrm{Hill}^{29}$ in the bidimensional case

$$
\text { if } \mathrm{n} \neq \mathrm{m} \quad\left(\mathbf{U}_{\mathrm{kn}} \mid \mathbf{U}_{\mathrm{km}}^{+}\right)=0 \quad \text { if } \quad \mathrm{n}=\mathrm{m} \quad \sigma_{\mathrm{km}}^{+}=-\sigma_{\mathrm{km}}^{\star} .
$$

\section{Optimal perturbation at large time}

The expansion of a perturbation, in the set of eigenfunctions, has been adressed by Salwen and Grosh ${ }^{37}$ for the Orr-Sommerfeld equation. We generalize here, their results, to the linearized Navier-Stokes equations.

If the eigenfunctions (A9) form a complete set, then for any time, a particular perturbation solution of Eq. (A1) may be expanded as a linear combination of those eigenfunctions

$$
\tilde{\mathbf{U}}(x, y, t)=\sum_{\mathrm{n}} A_{\mathrm{kn}}(t) \mathbf{U}_{\mathrm{kn}}(x, y) .
$$

The coefficient $A_{\mathrm{km}}(t)$ is evaluated using the biorthogonality condition (A13)

$$
\left(\tilde{\mathbf{U}}(x, y, t) \mid \mathbf{U}_{\mathrm{km}}^{+}(x, y)\right)=A_{\mathrm{km}}^{\star}(t)\left(\mathbf{U}_{\mathrm{km}}(x, y) \mid \mathbf{U}_{\mathrm{km}}^{+}(x, y)\right) .
$$

Moreover, $\tilde{\mathbf{U}}(x, y, t) \mathrm{e}^{\mathrm{ikz}}$ is a particular solution of the direct linearized Navier-Stokes operator (A1) and $U_{\mathrm{km}}^{+}(\mathrm{x}, \mathrm{y}) \mathrm{e}^{\mathrm{ikz}}$ $\mathrm{e}^{-\sigma_{\mathrm{km}}^{\star}}$ (with Eq. (A13) a particular solution of the adjoint linearized operator (A4); Eq. (A7) holds and yields to

$$
\frac{\partial}{\partial t}\left(\tilde{\mathbf{U}}(x, y, t) \mid \mathbf{U}_{\mathrm{km}}^{+}(\mathrm{x}, \mathrm{y}) \mathrm{e}^{-\sigma_{\mathrm{km}}^{\star} \mathrm{t}}\right)=0 .
$$

The amplitude $A_{\mathrm{km}}(t)$ is then solution to the following equation:

$$
\begin{aligned}
& -\sigma_{\mathrm{km}}^{\star} e^{-\sigma_{\mathrm{km}}^{\star} t} A_{\mathrm{km}}^{\star}(t)\left(\mathbf{U}_{\mathrm{km}}(\mathrm{x}, \mathrm{y}) \mid \mathbf{U}_{\mathrm{km}}^{+}(\mathrm{x}, \mathrm{y})\right) \\
& +\frac{\partial \mathrm{A}_{\mathrm{km}}^{\star}(\mathrm{t})}{\partial \mathrm{t}} \mathrm{e}^{-\sigma_{\mathrm{km}}^{\star}}\left(\mathbf{U}_{\mathrm{km}}(\mathrm{x}, \mathrm{y}) \mid \mathbf{U}_{\mathrm{km}}^{+}(\mathrm{x}, \mathrm{y})\right)=0,
\end{aligned}
$$

which gives

$$
A_{\mathrm{km}}(t)=e^{\sigma_{\mathrm{km}} t} A_{\mathrm{km}}(0)=e^{\sigma_{\mathrm{km}} t} \frac{\left(\tilde{\mathbf{U}}(x, y, 0) \mid \mathbf{U}_{\mathrm{km}}^{+}(\mathrm{x}, \mathrm{y})\right)}{\left(\mathbf{U}_{\mathrm{km}}(\mathrm{x}, \mathrm{y}) \mid \mathbf{U}_{\mathrm{km}}^{+}(\mathrm{x}, \mathrm{y})\right)},
$$

the value of the initial amplitude $A_{\mathrm{km}}(0)$ is obtained setting $t=0$ in Eq. (A15). We look for the initial condition, $\tilde{\mathbf{U}}(x, y, 0) e^{i \mathrm{kz}}$ which maximizes the energy $E(t)$ Eq. (11) at large time.

$$
\ln \mathrm{G}(\mathrm{t})=\max _{\tilde{\mathbf{U}}(x, y, 0)} \ln \frac{(\tilde{\mathbf{U}}(x, y, t) \mid \tilde{\mathbf{U}}(x, y, t))}{(\tilde{\mathbf{U}}(x, y, 0) \mid \tilde{\mathbf{U}}(x, y, 0))} .
$$

For a sufficiently large time, $\tilde{\mathbf{U}}(x, y, t)$ Eq. (A14) tends towards the most unstable eigenmode $A_{\mathrm{km}}(t) \mathbf{U}_{\mathrm{km}}(x, y)$. Thus, the amplification energy factor becomes

$$
\ln \mathrm{G}(\mathrm{t})=\max _{\tilde{\mathbf{U}}(x, y, 0)} \ln \left(\left|A_{\mathrm{km}}(t)\right|^{2} \frac{\left(\mathbf{U}_{\mathrm{km}}(\mathrm{x}, \mathrm{y}) \mid \mathbf{U}_{\mathrm{km}}(\mathrm{x}, \mathrm{y})\right)}{(\tilde{\mathbf{U}}(\mathrm{x}, \mathrm{y}, 0) \mid \tilde{\mathbf{U}}(\mathrm{x}, \mathrm{y}, 0))}\right),
$$

with Eq. (A18) we obtain

$$
\operatorname{lnG}(\mathrm{t})=\max _{\tilde{\mathbf{U}}(x, y, 0)} \ln \left(\frac{\left\|\mathbf{U}_{\mathrm{km}}(\mathrm{x}, \mathrm{y})\right\|^{2} \cdot\left\|\mathbf{U}_{\mathrm{km}}^{+}(\mathrm{x}, \mathrm{y})\right\|^{2} \cdot\|\tilde{\mathbf{U}}(\mathrm{x}, \mathrm{y}, 0)\|^{2} \cos ^{2} \alpha}{\|\tilde{\mathbf{U}}(\mathrm{x}, \mathrm{y}, 0)\|^{2} \cdot\left(\mathbf{U}_{\mathrm{km}}(\mathrm{x}, \mathrm{y}) \mid \mathbf{U}_{\mathrm{km}}^{+}(\mathrm{x}, \mathrm{y})\right)^{2}}\right)+2 \operatorname{Re}\left(\sigma_{\mathrm{km}}\right) \mathrm{t}
$$

with II.II, the nom associated with (.I.) and $\alpha$ the angle defined by

$$
\cos \alpha=\frac{\left(\tilde{\mathbf{U}}(x, y, 0) \mid \mathbf{U}_{\mathrm{km}}^{+}(x, y)\right)}{\left\|\mathbf{U}_{\mathrm{km}}^{+}(x, y)\right\| \cdot\|\cdot\| \mathbf{\mathbf { U }}(x, y, 0) \|} .
$$

The gain at large time is maximum for $\cos ^{2} \alpha=1$, thus for an initial condition colinear to the adjoint eigenmode. Denoting $\left.\mathbf{U}_{1}=\mathbf{U}_{\mathrm{km}}(x, y)\right) /\left\|\mathbf{U}_{\mathrm{km}}(x, y)\right\|$ and $U_{1}^{+}=U_{\mathrm{km}}^{+}(x, y) /\left\|U_{\mathrm{km}}^{+}(x, y)\right\|$, for simplicity, the optimal gain at large time is

$$
\ln \mathrm{G}(\mathrm{t})=\ln \left(\frac{1}{\left(\mathbf{U}_{1} \mid \mathbf{U}_{1}^{+}\right)^{2}}\right)+2 \operatorname{Re}\left(\sigma_{\mathrm{km}}\right) \mathrm{t}
$$

${ }^{1}$ P. G. Drazin and W. H. Reid, Hydrodynamic Stability (Cambridge University Press, Cambridge England, 1981).

${ }^{2}$ C. D. Winant and F. K. Browand, "Vortex pairing: The mechanism of turbulent mixing-layer growth at moderate Reynolds number," J. Fluid Mech. 63, 237 (1974).

${ }^{3}$ L. P. Bernal and A. Roshko, "Streamwise vortex structure in plane mixing layer," J. Fluid Mech. 170, 499 (1986).

${ }^{4}$ A. C. Robinson and P. G. Saffman, "Three-dimensional stability of an elliptical vortex in a straining field,” J. Fluid Mech. 142, 451 (1984). 
${ }^{5}$ E. Meiburg and J. C. Lasheras, "Experimental and numerical investigation of the three-dimensional transition in plane wakes," J. Fluid Mech. 190, 1 (1987).

${ }^{6}$ J. C. Lasheras and E. Meiburg, "Three dimensional vorticity modes in the wake of a flat plate," Phys. Fluids A 2(3), 371 (1990).

${ }^{7}$ C. H. K. Williamson, “Three-dimensional wake transition," J. Fluid Mech. 328, 345 (1994).

${ }^{8}$ C. H. K. Williamson, "Vortex dynamics in the cylinder wake," Ann. Rev. Fluid Mech. 28, 477 (1996).

${ }^{9}$ D. Barkley and R. D. Henderson, "Three-dimensional Floquet stability analysis of the wake of a circular cylinder," J. Fluid Mech. 322, 215 (1996).

${ }^{10}$ S. Julien, J. C. Lasheras, and J.-M. Chomaz, "Three-dimensional instability and vorticity patterns in the wake of a flat plate," J. Fluid Mech. 479, 155 (2003).

${ }^{11}$ R. R. Kerswell, "Elliptical instability," Annu. Rev. Fluid Mech. 34, 1 (2002).

${ }^{12}$ R. R. Lagnado, N. Phan-Thien, and L. G. Leal, "The stability of twodimensional linear flows," Phys. Fluids 27, 1094 (1984).

${ }^{13}$ A. D. D. Craik and W. O. Criminale, "Evolution of wavelike disturbances in shear flows: A class of exact solutions of the Navier-Stokes equations," Proc. R. Soc. London, Ser. A 406, 13 (1986).

${ }^{14}$ C. P. Caulfield and R. R. Kerswell, "The nonlinear development of threedimensional disturbances at hyperbolic stagnation points: A model of the braid region in mixing layers," Phys. Fluids 12(5), 1032 (2000).

${ }^{15}$ S. P. Sutherland and W. Peltier, "Turbulence transition and internal wave generation in density stratified jets," Phys. Fluids 6, 1276 (1994).

${ }^{16} \mathrm{~S}$. Julien, S. Ortiz, and J. M. Chomaz, "Secondary instability mechanisms in the wake of a flat plate," Eur. J. Mech. 23/1, 157 (2004).

${ }^{17}$ P. Luchini, "Reynolds-number-independant instability of the boundary layer over a flat surface: Optimal perturbations," J. Fluid Mech. 404, 289 (2000).

${ }^{18}$ B. F. Farrell and P. J. Ioannou, "Optimal excitation of three-dimensional perturbations in viscous constant shear flow," Phys. Fluids 5(6), 1390 (1993).

${ }^{19}$ A. Antkowiak and P. Brancher, "On vortex rings around vortices: An optimal mechanism,” J. Fluid Mech. 578, 295 (2007).

${ }^{20}$ S. Lin and G. Corcos, "The mixing layer: Deterministic models of turbulent flows. Part 3. The effect of plane strain on the dynamics of streamwise vortices," J. Fluid Mech. 141, 139 (1984).
${ }^{21}$ J. C. Neu, "The dynamics of stretched vortices," J. Fluid Mech. 143, 253 (1984).

${ }^{22}$ J. M. Chomaz, "Global instabilities in spatially developing flows: Nonnormality and nonlinearity,” Annu. Rev. Fluid. Mech. 37, 357 (2005).

${ }^{23}$ A. A. Monkewitz, "The absolute and convective nature of instability in two-dimensional wakes at low Reynolds numbers," Phys. Fluids 31, 999 (1988).

${ }^{24} \mathrm{P}$. Brancher, J.-M. Chomaz, and P. Huerre. "Direct numerical simulations of round jets: Vortex induction and side-jets," Phys. Fluids 6(5), 1768 (1994).

${ }^{25}$ W. S. Edwards, L. S. Tuckerman, R. A. Friesner, and D. C. Sorensen, "Krylov methods for the incompressible Navier-Stokes equations," J. Comput. Phys. 110, 82 (1994).

${ }^{26}$ F. Marques, J. M. Lopez, and H.-M. Blackburn, "Bifurcation in systems with Z2 spatio-temporal and O(2) spatial symmetry," Physica D 189, 247 (2004).

${ }^{27}$ P. G. Potylitsin and W. R. Peltier, "Stratification effects on the stability of columnar vortices on the f-plane," J. Fluid Mech. 355, 45 (1998).

${ }^{28}$ M. J. Landman and P. J. Saffman, "The three-dimensional instability of strained vortices in a viscous fluid," Phys. Fluids 30, 2339 (1987).

${ }^{29}$ D. C. Hill, "Adjoint systems and their role in boundary layer receptivity," J. Fluid Mech. 292, 183 (1995).

${ }^{30} \mathrm{P}$. Corbett and A. Bottaro, "Optimal perturbations for boundary layers subject to stream-wise pressure gradient," Phys. Fluids 12, 120 (1999).

${ }^{31} \mathrm{G}$. Corcos and S. Lin, "The mixing layer: Deterministic models of a turbulent flow. Part 2. The origin of the three-dimensional motion," J. Fluid Mech. 139, 67 (1984).

${ }^{32}$ A. Lifschitz and E. Hameiri, "Local stability conditions in fluid dynamics," Phys. Fluids. 3, 2644 (1991); Eur. J. Mech. 22, 411 (2003).

${ }^{33}$ C. Donnadieu, S. Ortiz, J.-M. Chomaz, and P. Billant, "Three-dimensional instabilities and transient growth of a counter-rotating vortex pair," Phys. Fluids 21, 094102 (2009).

${ }^{34}$ B. F. Farrell, "Optimal excitation of perturbation in viscous shear flows," Phys. Fluids A 31, 2093 (1988).

${ }^{35}$ E. L. Ince, Ordinary Differential Equations (Cambridge university Press, Cambridge, England, 1944).

${ }^{36} \mathrm{H}$. Salwen, "Expansions in spatial or temporal eigenmodes of the linearized Navier-Stokes equations," Bull. Am. Phys. Soc. 24, 74 (1979).

${ }^{37} \mathrm{H}$. Salwen and C. E. Grosch, "The continuous spectrum of the OrrSommerfeld equation. Part. 2. Eigenfunction expansions," J. Fluid Mech. 104, 445 (1981). 